\title{
SELF-SIMILAR BEHAVIOUR FOR NONCOMPACTLY SUPPORTED SOLUTIONS OF THE LSW MODEL
}

\author{
J. J. L. Velázquez ${ }^{1}$
}

\begin{abstract}
In this paper a method of approximating the classical LSW model near self-similar solutions for initial data with infinite support is developed. The resulting model is an integrodifferential equation having two time scales that can be studied using multiple scale methods. The analysis provides a detailed description of the precise manner in which the characteristics "leak" through the critical radius associated to the self-similar solutions. The analysis in this paper precises the meaning of the iterated logarithmic asymptotics in the dynamics of the LSW model that were already obtained in the original Lifshitz-Slyozov paper. Examples of noncompactly supported solutions of the LSW model that do not behave in self-similar manner are also given.
\end{abstract}

Keywords: LSW model, Ostwald ripening, Selfsimilar behaviour, Schwartzian derivative, Integral equations, Multiple scales analysis.

\section{INTRODUCTION}

This paper develops a mathematical formalism that allows to describe noncompactly supported solutions of the Lifshitz-Slyozov-Wagner model that behave in a self-similar manner for long times.

The Lifshitz-Slyozov-Wagner model (LSW) is the following nonlocal system of differential equations:

$$
\begin{aligned}
\frac{\partial f(R, t)}{\partial t}+\frac{\partial}{\partial R}\left(\left(-\frac{1}{R^{2}}+\frac{\Delta(t)}{R}\right) f(R, t)\right) & =0, t>0, R>0 \\
f(R, 0) & =f_{0}(R) \geq 0, \quad R>0 \\
\Delta(t) & =\frac{\int_{0}^{\infty} f(R, t) d R}{\int_{0}^{\infty} R f(R, t) d R}
\end{aligned}
$$

These equations are a classical model that describes the last stage of a phase transition process (Ostwald ripening) for which the the small volume fraction occupied by the particles is preserved (cf. $[5,18])$. Rigorous derivations of this system using homogenization techniques that take as starting point the Mullins-Sekerka free boundary problem have been obtained in different scaling limits in [3], [7], [8], $[12]$.

It is well known that the system (1)-(3) has a family of explicit self-similar solutions with the form (cf. $[5,18])$ :

$$
f(R, t)=\frac{1}{t^{4 / 3}} \Phi_{\alpha}(\rho), \rho=\frac{R}{t^{1 / 3}}
$$

where $\alpha \in(0, \infty]$. The self-similar solutions (4) are compactly supported in the variable $\rho$. For each given value of the volume fraction $\int_{0}^{\infty} \frac{4 \pi R^{3}}{3} f(R, t) d R$ the self-similar solutions are are uniquely characterized by means of their asymptotic behaviour near the maximum value of $\rho$. These asymptotics are given by:

$$
\Phi_{\beta}(\rho) \sim L_{\beta}\left(\rho_{\max }-\rho\right)^{\beta} \text { as } \rho \rightarrow \rho_{\max }^{-}
$$

\footnotetext{
${ }^{1}$ Departamento de Matemática Aplicada. Facultad de Matemáticas. Universidad Complutense. Madrid 28040. Spain.
} 
where $\beta>-1, \rho_{\max } \equiv \rho_{\max }(\beta), L_{\beta}>0$. On the other hand:

$$
\Phi_{\infty}(\rho) \sim L_{\infty}\left(\rho_{\max }-\rho\right)^{-1 / 3} \exp \left[-\frac{\left(\frac{3}{2}\right)^{2 / 3}}{\left(\rho_{\max }-\rho\right)}\right] \text { as } \quad \rho \rightarrow \rho_{\max }^{-}
$$

where $\rho_{\max } \equiv\left(\frac{3}{2}\right)^{1 / 3}$ and $L_{\infty}>0$. The numerical constants $L_{\beta}, L_{\infty}$ are proportional to the volume fraction filled by the particles.

Global well-posedness of the system (1)-(3) for compactly supported initial data has been proved in [10]. On the other hand, it has been rigorously proved in [9] that the initial data $f_{0}(R)$ must satisfy very stringent assumptions if the corresponding solution $f(R, t)$ behaves as one of the self-similar solutions (4) as $t \rightarrow \infty$ (see also [6] for a related formal study as well as [1] for an analogous study in a simplified model). Analogous results that include also some necessary conditions on compactly supported initial data that yield the asymptotics (6) can be found in [13], [14]. In an informal way the results in [9], [13], [14] can be formulated as saying that compactly supported initial initial data $f_{0}(R)$ for which the corresponding solutions behave asymptotically as $t \rightarrow \infty$ as (5)), (6) must behave near the maximum radius in the same way as these functions. Numerical studies of the asymptotics of the solutions of (1)-(3) can be found in [2].

The results of the papers [9], [13], [14] are valid only for compactly supported initial data, but not similar results have been derived for noncompactly supported initial data. The goal of this paper is to develop a theory that describes self-similar asymptotics for (1)-(3) at the level of formal asymptotics.

In [15] a formal asymptotic expansion for some particular noncompactly supported solutions of the LSW model that decay exponentially as $R \rightarrow \infty$ has been obtained. In a strict sense the results in [15] were derived for the Becker-Döring system of equations. Nevertheless the only role played in that paper by the Becker-Döring part of the equation is to tranform a compactly supported initial data in a noncompactly supported funtion that decreases exponentially for large radii. Besides this effect the analysis in [15] applies without relevant changes to the LSW system. A relevant feature of the analysis in [15] was a detailed analysis of some series that arise in the description of $\Delta(t)$ in (1) having the form:

$$
\Delta(t) \sim \frac{c_{0}}{t^{1 / 3}}\left[1+c_{1}\left(\frac{1}{(\log (t))^{2}}+\frac{1}{(\log (t))^{2}(\log (\log (t)))^{2}}+\ldots\right)\right]
$$

for suitable constants $c_{0}, c_{1}$.

Similar series had been originally obtained in seminal paper [5]. The analysis of this paper will provide an explanation for the onset of the series (7) for noncompactly supported solutions of (1)-(3). On the other hand, the idea of using the Becker-Döring system in order to find a selection mechanism for "correct" self-similar behaviour of the LSW model has been introduced also in [6]. We point out that the series (7) arise in the study of the "leaking" of the characteristic curves near the critical radius. It was already noticed in the original paper [5] that such "leaking" of characteristics plays a crucial role in the study of solutions behaving in a self-similar manner for long times. The main goal of this paper is to understand such "leaking" of characteristics in a detailed way.

A key point in the study made in the paper(s) $[13,14]$ was to approximate the dynamics of the compactly supported solutions of the LSW system (1)-(3) that remain close to self-similar solutions for long times by means of some integral equations. In this paper we will derive also some integral equations that approximate the LSW dynamics near self-similar solutions for noncompactly supported initial data. There are, however, several differences between the integral equations in $[13,14]$ and the ones in this paper. Namely, the integral equations that arise for compactly supported initial data are of convolution type and have only one characteristic time scale. On the contrary, the equations derived in this paper for noncompactly supported initial data have two characteristic time scales as $t \rightarrow \infty$. Nevertheless, the solutions of these equations can be approximated for long times using multiple scales techniques. 
The plan of the paper is the following. In Section 2 we recall some basic facts about the self-similar solutions of the LSW model. In Section 3 we collect some of the basic ideas used in [13, 14] on the analysis of the long time asymptotics for the solutions of the LSW model for compactly supported initial data that. In Section 4 that contains the main results of this paper we study in detail the asymptotic behaviour of the characteristic curves associated to the LSW system yielding self-similar behaviour for the solutions of the problem. Some technical results are collected in several Appendixes at the end of the paper.

\section{PRELIMINARY RESULTS. SELF-SIMILAR SOLUTIONS}

Some computations will become simpler using as independent variable the volume of the particles $v$ instead of their radii, and replacing the particle density by the cumulative volume distribution. We define $\bar{f}(v, t)$ by means of:

$$
\begin{aligned}
v & =R^{3}, \bar{t}=3 t \\
f(R, t) d R & =\bar{f}(v, \bar{t}) d v
\end{aligned}
$$

whence $\bar{f}(v, \bar{t})=\frac{1}{3 v^{2 / 3}} f\left(v^{1 / 3}, t\right)$. We now introduce the cumulative volume distribution

$$
F(v, \bar{t}) \equiv \int_{v}^{\infty} \bar{f}(\xi, \bar{t}) d \xi
$$

Using (1)-(3) it follows that $F(v, \bar{t})$ satisfies:

$$
\begin{aligned}
\frac{\partial F(v, \bar{t})}{\partial \bar{t}}+\left(-1+\Delta(\bar{t}) v^{1 / 3}\right) \frac{\partial F(v, \bar{t})}{\partial v} & =0, \bar{t}>0, v>0 \\
F(v, 0) & =F_{0}(v) \equiv \int_{v}^{\infty} \bar{f}_{0}(v) d \xi, v>0 \\
\Delta(\bar{t}) & =\frac{3 F\left(0^{+}, \bar{t}\right)}{\int_{0}^{\infty} v^{-2 / 3} F(v, \bar{t}) d v}
\end{aligned}
$$

Due to the positivity of $f$ it follows that $F(\cdot, \bar{t})$ is a decreasing function. The conservation of the total volume of the particles for the LSW model is:

$$
\frac{d}{d \bar{t}}\left(\int_{0}^{\infty} F(v, \bar{t}) d v\right)=0
$$

We define a set of self-similar variables by means of:

$$
F(v, \bar{t})=\frac{G(W, \tau)}{\bar{t}}, W=\frac{2 v}{t}, \tau=\log (\bar{t})
$$

In these new set of variables (9)-(11) becomes:

$$
\begin{aligned}
\frac{\partial G}{\partial \tau}+\left(-2+3 \lambda(\tau) W^{1 / 3}-W\right) \frac{\partial G}{\partial W} & =G \\
\lambda(\tau) & =\frac{2 G\left(0^{+}, \tau\right)}{\int_{0}^{\infty} W^{-2 / 3} G(W, \tau) d W} \\
G(W, 0) & =G_{0}(W)
\end{aligned}
$$


The self-similar solutions of the LSW model are the steady states of of the system of equations (14)-(16). They are given by the formulae:

$$
G_{s}\left(W ; \lambda_{0}\right)=\exp \left(-\int_{0}^{W} \frac{d \xi}{2-3 \lambda_{0} \xi^{1 / 3}+\xi}\right) \quad, 0 \leq W<W_{0}, G_{s}(W)=0 \text { if } W \geq W_{0}
$$

where $\lambda_{0} \geq 1$, and $W_{0}$ is the smallest positive root of the equation:

$$
2-3 \lambda_{0}\left(W_{0}\right)^{1 / 3}+W_{0}=0
$$

Since $W_{0} \leq 1$, for any $\lambda_{0} \geq 1$ it follows that the solution from the family (17) having maximal support is the corresponding to $\lambda_{0}=1$. Since that particular solution will be used repeatedly in the following we write it separatedly by convenience

$$
G_{s}(W)=\exp \left(-\int_{0}^{W} \frac{d \xi}{2-3 \xi^{1 / 3}+\xi}\right), 0 \leq W<1, G_{s}(W)=0 \text { if } W \geq 1 .
$$

\section{LSW DYNAMICS NEAR SELF-SIMILAR SOLUTIONS: COM- PACTLY SUPPORTED CASE}

In this Section we briefly recall some results concerning long time self-similar behaviours for compactly supported solutions of the LSW model that have been proved in [13, 14]. Some of the ideas used in this Section to describe the asymptotics of compactly supported initial data will be used later in the analysis of noncompactly supported initial data.

In the compactly supported case it is convenient to use a different set of self-similar variables. Suppose that the support of $F(v, \bar{t})$ is the interval $\left[0, v_{\max }(\bar{t})\right]$. We define a new set of adimensional variables by means of:

$$
\begin{aligned}
G(V, \tau) & =v_{\max }(t) F(v, \bar{t}) \\
V & =\frac{v}{v_{\max }(t)} \\
\tau & =\log \left(\frac{v_{\max }(\bar{t})}{v_{\max }(0)}\right)
\end{aligned}
$$

Using this new set of variables (9)-(11) become:

$$
\begin{aligned}
G(V, \tau) & =\frac{\partial G(V, \tau)}{\partial \tau}+\left(\left(V^{1 / 3}-V\right)+\kappa(\tau)\left(V^{1 / 3}-1\right)\right) \frac{\partial G(V, \tau)}{\partial V} \\
\frac{1}{\kappa(\tau)} & =\frac{d v_{\max }(t)}{d t}=-1+\frac{3 G\left(0^{+}, \tau\right)}{\int_{0}^{\infty}(V)^{-2 / 3} G(V, \tau) d V} \\
G(V, 0) & =G_{0}(V)=v_{\max }(0) F_{0}\left(v_{\max }(0) V\right)
\end{aligned}
$$

and the self-similar solutions in (17) would be given as:

$$
\begin{aligned}
G_{s}(V) & =C \exp \left(-\int_{0}^{V} \frac{d \xi}{\kappa_{0}\left(1-\xi^{1 / 3}\right)+\left(\xi-\xi^{1 / 3}\right)}\right), V>0 \\
\kappa(\tau) & =\kappa_{0}
\end{aligned}
$$

where $\kappa_{0} \geq 2$. The main advantage of using the variables (20)-(22) instead of (13) is that the support of the solutions becomes the fixed interval $[0,1]$. 
The asymptotics of the self-similar solutions near the maximum radius is:

$$
\begin{aligned}
& G_{s}(V) \sim C B\left(\kappa_{0}\right)(1-V)^{\alpha}, \text { as } V \rightarrow 1^{-}, \alpha=\frac{3}{\kappa_{0}-2} \text { if } \kappa_{0}>2 \\
& G_{s}(V) \sim A C(1-V)^{5 / 3} e^{-\frac{3}{(1-V)}}, \text { as } V \rightarrow 1^{-} \text {if } \kappa_{0}=2
\end{aligned}
$$

where $B\left(\kappa_{0}\right)>0, A>0$ can be explicitly computed by means of some integral formulae whose precise form is not particularly relevant for the purposes of this paper.

In order to describe the dynamics of compactly supported solutions of the LSW model near the self-similar solution characterized by the parameter $\kappa_{0}$ it is convenient to use the new variable:

$$
Z=\int_{0}^{V} \frac{d \xi}{\kappa_{0}\left(1-\xi^{1 / 3}\right)+\left(\xi-\xi^{1 / 3}\right)}
$$

Using this variable the system (23), (24) becomes:

$$
\begin{aligned}
G & =\frac{\partial G}{\partial \tau}+(-1-\beta(\tau) H(Z)) \frac{\partial G}{\partial Z} \\
\beta(\tau) & =\Phi\left(\int_{0}^{1}(V)^{-2 / 3}\left(\frac{G(Z, \tau)}{G\left(0^{+}, \tau\right)}-e^{-Z}\right) d V\right) \\
G(Z, 0) & =G_{0}(Z)
\end{aligned}
$$

where

$$
\begin{aligned}
& \beta(\tau) \equiv \kappa(\tau)-\kappa_{0} \\
& \Phi(x) \equiv \frac{\left(\kappa_{0}+1\right)^{2} \kappa_{0}}{9} \frac{x}{\kappa_{0}-\frac{\left(\kappa_{0}-2\right)\left(\kappa_{0}+1\right)}{9} x}
\end{aligned}
$$

and:

$$
H(Z)=\frac{\left(1-V^{1 / 3}\right)}{\left(V-V^{1 / 3}\right)+\kappa_{0}\left(1-V^{1 / 3}\right)}=\frac{1}{\left(\kappa_{0}-(V)^{2 / 3}-V^{1 / 3}\right)}
$$

with $V$ and $Z$ related by means of (30). The self-similar solution (26) becomes then

$$
G_{s}(Z)=C e^{-Z}
$$

We now study in a separate manner the dynamics of the LSW model near the self-similar solutions with $\kappa_{0}>2$ and $\kappa_{0}=2$ respectively.

\subsection{Dynamics of the LSW model near self-similar solutions with $\kappa_{0}>2$.}

As it was proved in [9] that an arbitrary initial data $G_{0}(Z)$ does not yield a solution $G(Z, \tau)$ behaving as the self-similar solution (35) as $\tau \rightarrow \infty$. We recall the necessary condition that $G_{0}(Z)$ must satisfy in order to have self-similar behaviour.

\subsubsection{Necessary conditions for self-similar behaviour.}

Suppose that $G(Z, \tau)$ behaves in a self-similar manner as $\tau \rightarrow \infty$. i.e.

$$
\lim _{\tau \rightarrow \infty} \frac{G(Z, \tau)}{G\left(0^{+}, \tau\right)}=e^{-Z}
$$


Due to (32) it follows that:

$$
\lim _{\tau \rightarrow \infty} \beta(\tau)=0
$$

Integrating (31), (33) using characteristics we obtain

$$
\frac{G(Z, \tau)}{G\left(0^{+}, \tau\right)}=\frac{G_{0}\left(Z_{0}\right)}{G_{0}\left(\bar{Z}_{0}\right)}
$$

where from now on we denote as $Z\left(s, Z_{0}\right)$ the solution of the characteristic equation

$$
\begin{aligned}
Z_{s} & =(-1-\beta(s) H(Z)) \\
Z\left(0, Z_{0}\right) & =Z_{0}
\end{aligned}
$$

with boundary data

$$
Z\left(\tau, Z_{0}\right)=Z, Z\left(\tau^{-}, \bar{Z}_{0}\right)=0^{+}
$$

By assumption, $Z_{0}=Z_{0}(\tau, Z), \bar{Z}_{0}=\bar{Z}_{0}(\tau)$. Moreover, the function $Z_{0}(\tau, \cdot)$ is one-one for each fixed value of $\tau$.

Differentiating (39), (40) with respect to $Z_{0}$ we obtain:

$$
\begin{aligned}
\left(\frac{\partial Z}{\partial Z_{0}}\right)_{s} & =-\beta(s) H^{\prime}\left(Z\left(s, Z_{0}\right)\right) \frac{\partial Z}{\partial Z_{0}} \\
\frac{\partial Z}{\partial Z_{0}}\left(0, Z_{0}\right) & =1
\end{aligned}
$$

On the other hand, combining (30), (34) it follows that, for $\kappa_{0}>2$ :

$$
\begin{aligned}
H(Z) & =\frac{1}{\kappa_{0}-2}+O\left(e^{-a Z}\right) \text { as } Z \rightarrow \infty \\
H^{\prime}(Z) & =O\left(e^{-a Z}\right) \text { as } Z \rightarrow \infty
\end{aligned}
$$

for some $a>0$.

Integrating (41), (42) we arrive at

$$
\frac{\partial Z}{\partial Z_{0}}=\exp \left(-\int_{0}^{\tau} \beta(\xi) H\left(Z\left(\xi, Z_{0}\right)\right) d \xi\right)
$$

On the other hand, using (37) it follows that $Z\left(s, Z_{0}\right)$ decreases at least linearly for $s$ large enough. In particular $Z_{0}(\tau, Z)$ grows linearly on $\tau$ as $\tau \rightarrow \infty$ for $Z$ in bounded sets. Using this as well as the exponential decay (44) it follows that:

$$
\lim _{\tau \rightarrow \infty}\left(\int_{0}^{\tau} \beta(\xi) H\left(Z\left(\xi, Z_{0}\right)\right) d \xi\right)=0
$$

uniformly for $Z$ in bounded sets. Then:

$$
\frac{\partial Z_{0}(\tau, Z)}{\partial Z} \rightarrow 1
$$

as $\tau \rightarrow \infty$ uniformly on compact sets of $Z$. Therefore, integrating with respect to $Z$ we obtain:

$$
Z=\left(Z_{0}-\bar{Z}_{0}\right)(1+\delta(\tau, Z))
$$

where $\lim _{\tau \rightarrow \infty} \delta(\tau, Z)=0$ uniformly on compact sets of $Z$. Let us write $\zeta=Z_{0}-\bar{Z}_{0}$. Combining (36), (38) and (45) and using that $\bar{Z}_{0} \rightarrow \infty$ as $\tau \rightarrow \infty$ we finally arrive at:

$$
\lim _{\bar{Z}_{0} \rightarrow \infty} \frac{G_{0}\left(\bar{Z}_{0}+\zeta\right)}{G_{0}\left(\bar{Z}_{0}\right)}=e^{-\zeta}
$$

uniformly on compact sets of $\zeta$.

Condition (46) is the necessary condition that was obtained in [9] and that must be assumed for the initial data $G_{0}(\cdot)$ in order to have self-similar behaviours for $G(Z, \tau)$ as $\tau \rightarrow \infty$ with $\kappa_{0}>2$. 


\subsubsection{Sufficient conditions for self-similar behaviour.}

We now proceed to describe the asymptotic behaviour of the solutions of (31)-(33) with initial data $G_{0}(\cdot)$ satisfying (46). It has been rigorously proved in [9], [13] that the corresponding solution behaves in a self-similar manner for long times under some additional assumptions. Our goal is to approximate the LSW dynamics near such self-similar behaviour, or more precisely, to find a linearization procedure of the LSW model that are close to self-similar.

Linearizing in (32) and using that due to $(24),(35) \frac{1}{\int_{0}^{1}(V)^{-2 / 3} e^{-Z} d V}=\frac{\kappa_{0}+1}{3}$, we arrive at:

$$
\beta(\tau)=\frac{\left(\kappa_{0}+1\right)^{2}}{9} \int_{0}^{1}(V)^{-2 / 3}\left(\frac{G(Z, \tau)}{G\left(0^{+}, \tau\right)}-e^{-Z}\right) d V
$$

In order to compute $\frac{G(Z, \tau)}{G\left(0^{+}, \tau\right)}$ it is convenient to eliminate first the exponential dependence $e^{-Z}$ by means of the change of variables:

$$
G(Z, \tau)=S(Z, \tau) e^{-Z}
$$

Then, (31) implies that $S(Z, \tau)$ solves:

$$
\begin{aligned}
\frac{\partial S}{\partial \tau}-(1+\beta(\tau) H(Z)) \frac{\partial S}{\partial Z} & =-\beta(\tau) H(Z) S \\
S(Z, 0) & =S_{0}(Z) \equiv G_{0}(Z) e^{Z}
\end{aligned}
$$

Integrating by characteristics (49), (50) we obtain:

$$
\frac{S(Z, \tau)}{S\left(0^{+}, \tau\right)}=\frac{S_{0}\left(Z_{0}(\tau, Z)\right)}{S_{0}\left(Z_{0}\left(\tau, 0^{+}\right)\right)} e^{-\int_{0}^{\tau} \beta(s)\left[H\left(Z\left(s, Z_{0}(\tau, Z)\right)\right)-H\left(Z\left(s, Z_{0}\left(\tau, 0^{+}\right)\right)\right)\right] d s}
$$

Plugging (48) and (51) into (47) we arrive at:

$$
\begin{aligned}
& \beta(\tau) \\
& =\frac{\left(\kappa_{0}+1\right)^{2}}{9} \int_{0}^{1}(V)^{-2 / 3} e^{-Z}\left(\frac{S_{0}\left(Z_{0}(\tau, Z)\right)}{S_{0}\left(Z_{0}\left(\tau, 0^{+}\right)\right)}-1\right) e^{-\int_{0}^{\tau} \beta(s)\left[H\left(Z\left(s, Z_{0}(\tau, Z)\right)\right)-H\left(Z\left(s, Z_{0}\left(\tau, 0^{+}\right)\right)\right)\right] d s} d V \\
& +\frac{\left(\kappa_{0}+1\right)^{2}}{9} \int_{0}^{1}(V)^{-2 / 3} e^{-Z}\left[e^{-\int_{0}^{\tau} \beta(s)\left[H\left(Z\left(s, Z_{0}(\tau, Z)\right)\right)-H\left(Z\left(s, Z_{0}\left(\tau, 0^{+}\right)\right)\right)\right] d s}-1\right] d V
\end{aligned}
$$

The asymptotics (43) as well as the fact that $Z\left(s, Z_{0}(\tau, Z)\right)$ increases linearly on $(\tau-s)$ as $(\tau-s) \rightarrow$ $\infty$ imply that $\left[H\left(Z\left(s, Z_{0}(\tau, Z)\right)\right)-H\left(Z\left(s, Z_{0}\left(\tau, 0^{+}\right)\right)\right)\right]$might be exponentially bounded on $(\tau-s)$ for large values of this variable. Therefore, the integral term $\int_{0}^{\tau} \beta(s)\left[H\left(Z\left(s, Z_{0}(\tau, Z)\right)\right)-H\left(Z\left(s, Z_{0}\left(\tau, 0^{+}\right)\right)\right)\right] d s$ is small for small $\beta(\cdot)$. Linearizing in the last term in $(52)$ it then follows that:

$$
\beta(\tau)=-\int_{0}^{\tau} K(\tau, s) \beta(s) d s+\varepsilon(\tau)
$$

where

$$
K(\tau, s) \equiv \frac{\left(\kappa_{0}+1\right)^{2}}{9} \int_{0}^{1}(V)^{-2 / 3} e^{-Z}\left[H\left(Z\left(s, Z_{0}(\tau, Z)\right)\right)-H\left(Z\left(s, Z_{0}\left(\tau, 0^{+}\right)\right)\right)\right] d V
$$

and where $\varepsilon(\tau)$ is the first term on the right-hand side of (52) and might be bounded as:

$$
|\varepsilon(\tau)| \leq C \int_{0}^{1}(V)^{-2 / 3} e^{-Z}\left|\frac{S_{0}\left(Z_{0}(\tau, Z)\right)}{S_{0}\left(Z_{0}\left(\tau, 0^{+}\right)\right)}-1\right| d V
$$


The necessary condition for self-similar behaviour (46) combined with (48) yield:

$$
\lim _{\tau \rightarrow \infty} \varepsilon(\tau)=0
$$

On the other hand, since $\left[H\left(Z\left(s, Z_{0}(\tau, Z)\right)\right)-H\left(Z\left(s, Z_{0}\left(\tau, 0^{+}\right)\right)\right)\right]$decreases exponentially as $(\tau-s) \rightarrow$ $\infty$, we can compute an approximation of this function using that, to the leading order, for small $\beta(\cdot)$ :

$$
Z\left(s, Z_{0}(\tau, Z)\right) \sim Z+(\tau-s)
$$

Therefore:

$$
K(\tau, s) \sim K(\tau-s) \equiv \frac{\left(\kappa_{0}+1\right)^{2}}{9} \int_{0}^{1}(V)^{-2 / 3} e^{-Z}[H(Z+(\tau-s))-H(\tau-s)] d V
$$

and the integral equation for $\beta(\cdot)$ becomes the convolution equation:

$$
\beta(\tau)=-\int_{0}^{\tau} K(\tau-s) \beta(s) d s+\varepsilon(\tau)
$$

where $K(\cdot)$ is a positive, strictly decreasing function.

The integral equation (56) provides the sought-for approximation of the LSW model near the selfsimilar solutions (26) with $\kappa_{0}>2$. The analysis above has been made precise and fully rigorous in [13]. A similar equation has been found in [16]. The equation (56) is of convolution type and its behaviour can be obtained using either Laplace transforms as in [16] or just a direct analisis of the equation like in [13]. The final result derived there is that the solutions of the integral equation (56) satisfy

$$
\lim _{\tau \rightarrow \infty} \beta(\tau)=0
$$

Notice that in particular, that the study of the integral equation (56) provides a description of the asymptotics of $\beta(\tau)$ as well as of the $G(Z, \tau)$ in terms of the function $\varepsilon(\tau)$ that measures the distance between the initial data $G_{0}(Z)$ and the self-similar solutions of the LSW model.

\subsection{Dynamics of the LSW model near self-similar solutions with $\kappa_{0}=2$.}

We begin discussing, as in the case $\kappa_{0}>2$, necessary conditions for self-similar behaviour.

\subsubsection{Necessary conditions for self-similar behaviour.}

The class of initial data yielding self-similar behaviour as $\tau \rightarrow \infty$ is, in some sense, much wider that in the case $\kappa_{0}>2$.

In the case $\kappa_{0}=2$ the asymptotic behaviour of $H(Z)$ defined in (34) is:

$$
H(Z) \equiv \frac{Z}{3}+Q(Z) \sim \frac{Z}{3}+\frac{5}{9} \log (Z)+C+o(1) \text { as } Z \rightarrow \infty
$$

Differentiating (39), (40) we obtain:

$$
\begin{aligned}
\left(\frac{\partial Z}{\partial Z_{0}}\right)_{s} & =-\beta(s) H^{\prime}\left(Z\left(s, Z_{0}\right)\right) \frac{\partial Z}{\partial Z_{0}}=-\beta(s)\left[\frac{1}{3}+Q^{\prime}\left(Z\left(s, Z_{0}\right)\right)\right] \frac{\partial Z}{\partial Z_{0}} \\
\frac{\partial Z}{\partial Z_{0}}\left(0, Z_{0}\right) & =1
\end{aligned}
$$


whence:

$$
\frac{\partial Z}{\partial Z_{0}}\left(s, Z_{0}\right)=\frac{e^{-\frac{1}{3} \int_{0}^{s} \beta(\xi) d \xi}}{\psi(s, Z)}
$$

where:

$$
\psi(\tau, Z)=e^{\int_{0}^{\tau} \beta(\xi) Q^{\prime}\left(Z\left(\xi, Z_{0}\right)\right) d \xi}
$$

Formula (61) provides a decomposition of the stretching of the characteristics in the part due to the term $\frac{Z}{3}$ in (58) and in the part due to the logarithmic corrective term $Q(Z)$ that is the one given by (62). It turns out that the last part is basically constant for characteristics arriving to points $Z_{1}, Z_{2}$ that differ an amount of order one, or more precisely (cf. [14])

$$
\left|\frac{\psi(\tau, Z)-\psi(\tau, 0)}{\psi(\tau, 0)}\right|<<1 \text { as } \tau \rightarrow \infty
$$

Plugging this formula into (61) we arrive at:

$$
\frac{\partial Z}{\partial Z_{0}}\left(\tau, Z_{0}\right)=\frac{e^{-\frac{1}{3} \int_{0}^{\tau} \beta(\xi) d \xi}}{\psi(\tau, 0)}(1+o(1)) \text { as } \tau \rightarrow \infty
$$

uniformly on bounded sets of $Z$. Integrating (63) with respect to $Z_{0}$ we obtain:

$$
Z_{0}-\bar{Z}_{0}=\psi(\tau, 0) e^{\frac{1}{3} \int_{0}^{\tau} \beta(\xi) d \xi}(1+o(1)) Z \equiv m(\tau)(1+o(1)) Z \text { as } \tau \rightarrow \infty
$$

uniformly on bounded sets of $Z$, where $\bar{Z}_{0}$ has the same meaning as in Subsection 3.1.

Formula (61) yields the stretching of the characteristics. Notice that due to the linear growth of $H(Z)$ the stretching suffered by the characteristics depends much more strongly on $\beta(\tau)$ that in the case $\kappa_{0}>2$, (cf. (45)). As a consequence the class of initial data $G_{0}$ yielding self-similar behaviours in the case $\kappa_{0}=2$ is much larger.

Indeed, integrating (31), (33) by characteristics, we obtain (37). Since, by assumption $G(Z, \tau)$ behaves in a self-similar manner we obtain:

$$
\frac{G_{0}\left(\bar{Z}_{0}+m(\tau)(1+o(1)) Z\right)}{G_{0}\left(\bar{Z}_{0}\right)} \sim e^{-Z} \text { as } \tau \rightarrow \infty
$$

Since $\bar{Z}_{0}$ is an increasing function of $\tau$ we can write $m(\tau)=\lambda\left(\bar{Z}_{0}\right)$. It follows that:

$$
\lim _{\bar{Z}_{0} \rightarrow \infty} \frac{G_{0}\left(\bar{Z}_{0}+\lambda\left(\bar{Z}_{0}\right) Z\right)}{G_{0}\left(\bar{Z}_{0}\right)}=e^{-Z}
$$

uniformly on compact sets of $Z$.

Condition (66) is the necessary condition that must be assumed on the initial data $G_{0}$ in order to obtain long time self-similar behaviour with $\kappa_{0}=2$ for compactly supported initial data. This condition plays a role analogous to (46) in the case $\kappa_{0}>2$. A more detailed derivation of the condition (66) can be found in [14].

We will now prove by means of formal arguments that this condition is also sufficient for self-similar behaviour.

\subsubsection{Sufficient conditions for self-similar behaviour.}

Our next goal is to deduce a "linearized version" of the LSW model playing a role analogous to (56) in the case $\kappa_{0}>2$. 
In the case $\kappa_{0}=2$ the equation for $\beta(\cdot)$ (32) reduces, after integrating by characteristics (37) to:

$$
\beta(\tau)=\int_{0}^{1}(V)^{-2 / 3}\left(\frac{G_{0}\left(Z_{0}\right)}{G_{0}\left(\bar{Z}_{0}\right)}-e^{-Z}\right) d V
$$

We now need to approximate $Z_{0}-\bar{Z}_{0}$. This computation has been made in [14] where the following formula has been derived

$$
Z_{0}-\bar{Z}_{0}=f(\tau)\left[Z+\int_{0}^{\tau} \beta(s) K(\tau-s, Z) d s+\delta(\tau, Z)\right]
$$

where:

$$
\begin{aligned}
f(\tau) & =e^{\frac{1}{3} \int_{0}^{\tau} \beta(\xi) d \xi} \psi(\tau, a) \\
K(\tau, Z) & =\left[Q(Z+\tau)-Q(\tau)-Q^{\prime}(a+\tau) Z\right]
\end{aligned}
$$

where $a>0$ is a fixed, large number, and where $\lim _{\tau \rightarrow \infty} \delta(\tau, Z)=0$ uniformly on compact sets of $Z$.

Combining (66), (68) we obtain

$$
\frac{G_{0}\left(Z_{0}\right)}{G_{0}\left(\bar{Z}_{0}\right)} \sim e^{-\frac{f(\tau)}{\lambda\left(Z_{0}\right)}\left[Z+\int_{0}^{\tau} \beta(\xi) K(\tau-\xi, Z) d \xi+R(\tau, \xi)\right]}
$$

Since we are interested in solutions behaving in a self-similar manner as $\tau \rightarrow \infty$ we must assume that $\frac{f(\tau)}{\lambda\left(\bar{Z}_{0}\right)} \rightarrow 1$ as $\tau \rightarrow \infty$. Therefore we can linearize (71) to obtain

$$
\frac{G_{0}\left(Z_{0}\right)}{G_{0}\left(\bar{Z}_{0}\right)} \sim e^{-Z}\left[1-\left(\frac{f(\tau)}{\lambda\left(\bar{Z}_{0}\right)}-1\right) Z-\int_{0}^{\tau} \beta(s) K(\tau-s, Z) d s+\delta(\tau, Z)\right]
$$

Plugging this formula into (67) we obtain:

$$
\beta(\tau)=-\Gamma_{0}\left(\frac{f(\tau)}{\lambda\left(\bar{Z}_{0}\right)}-1\right)-\int_{0}^{\tau} \beta(s) \Phi(\tau-s) d s+v_{1}(\tau)
$$

where:

$$
\begin{gathered}
\Gamma_{0} \equiv \int_{0}^{1}(V)^{-2 / 3} e^{-Z} Z d V \\
\Phi(\tau) \equiv \int_{0}^{1}(V)^{-2 / 3} e^{-Z} K(\tau, Z) d V
\end{gathered}
$$

and where $v_{1}(\tau) \equiv \int_{0}^{1}(V)^{-2 / 3} e^{-Z} \delta(\tau, Z) d V$ satisfies

$$
\left|v_{1}(\tau)\right|<<\bar{\beta}(\tau) .
$$

From now on we will denote as $v_{k}(\tau), k=1,2, \ldots$ a generic function satisfying (75).

We need a relation between $f(\tau)$ and $\beta(\tau)$. Using (69) we arrive at:

$$
\beta(\tau)=3\left[\frac{f^{\prime}(\tau)}{f(\tau)}-\frac{\psi_{\tau}(\tau, a)}{\psi(\tau, a)}\right]
$$

The quotient $\frac{\psi_{\tau}(\tau, a)}{\psi(\tau, a)}$ can be computed differentiating (62), using (39) as well as the fact that $\frac{\frac{\partial Z\left(\xi, Z_{0}\right)}{\partial Z_{0}}}{\frac{\partial Z\left(\tau, Z_{0}\right)}{\partial Z_{0}}} \rightarrow$ 1 as $\tau \rightarrow \infty$. The resulting formula for $\beta(\tau)$ after some manipulations is

$$
\beta(\tau)=\frac{3 f^{\prime}(\tau)}{f(\tau)}-3 \int_{0}^{\tau} \beta(\xi) Q^{\prime \prime}(a+(\tau-\xi)) d \xi+v_{2}(\tau)
$$


In order to transform the problem $(72),(77)$ in a linear problem we introduce a new variable:

$$
\varphi(\tau) \equiv \frac{f(\tau)}{\lambda\left(\bar{Z}_{0}\right)}-1
$$

In the previous computations it has been assumed that $\varphi$ is small. Moreover, it turns out that under this assumption $\frac{\lambda^{\prime}\left(\bar{Z}_{0}\right)}{\lambda\left(\bar{Z}_{0}\right)} \frac{d \bar{Z}_{0}}{d \tau} \sim \lambda^{\prime}\left(\bar{Z}_{0}\right) \rightarrow 0$, where the last formula is a consequence of (66) (cf. [14]). Using this fact we might transform (77) into

$$
\begin{gathered}
\beta(\tau)=3 \varphi^{\prime}(\tau)-3 \int_{0}^{\tau} \beta(s) Q^{\prime \prime}(a+(\tau-s)) d s+v_{2}(\tau) \\
\beta(\tau)=-\Gamma_{0} \varphi(\tau)-\int_{0}^{\tau} \beta(s) \Phi(\tau-s) d s+v_{1}(\tau)
\end{gathered}
$$

The system (79), (80), provides a description of of the dynamics of the LSW system for solutions that are close to the self-similar solution with $\kappa_{*}=2$.

We define the fundamental solution for $(80),(79)$ as the solution of:

$$
\begin{aligned}
& \beta_{f}(\tau, \theta)=-\Gamma_{0} \varphi_{f}(\tau, \theta)-\int_{0}^{\tau} \beta_{f}(s, \theta) \Phi(\tau-s) d s+A \delta(\tau-\theta) \\
& \beta_{f}(\tau, \theta)=3 \varphi_{f}^{\prime}(\tau, \theta)-3 \int_{0}^{\tau} \beta_{f}(s, \theta) Q^{\prime \prime}(a+(\tau-s)) d s+B \delta(\tau-\theta)
\end{aligned}
$$

Since the integral operators are of convolution type this system can be solved using Laplace transforms. More precisely, the fundamental solution $\left(\beta_{f}(\tau, \theta), \varphi_{f}(\tau, \theta)\right)$ has been explicitly computed in [14] where it has been found that:

$$
\begin{aligned}
\beta_{f}(\tau, \theta) & =A \delta(\tau-\theta)+\beta_{r e g}(\tau, \theta) \\
\left|\beta_{r e g}(\tau, \theta)\right| & \leq \frac{C}{1+(\tau-\theta)^{\gamma}}, \tau \geq \theta \\
\left|\varphi_{f}(\tau, \theta)\right| & \leq \frac{C}{1+(\tau-\theta)^{\gamma}}, \tau \geq \theta
\end{aligned}
$$

with $1<\gamma<2$. We can then compute $\beta(\tau), \varphi(\tau)$ computing the convolution of $\beta_{f}(\tau, \theta), \varphi_{f}(\tau, \theta)$ and $v_{1}(\tau), v_{2}(\tau)$ respectively. Therefore

$$
|\beta(\tau)|+|\varphi(\tau)| \rightarrow 0 \text { as } \tau \rightarrow \infty
$$

We remark that in particular this result confirms "a posteriori" the assumptions made in the derivation of (80), (79) and it shows that the self-similar behaviour with $\kappa_{0}=2$ is indeed stable, under the assumption (66). We refer to [14] for details and precise formulations of the results.

\section{NONCOMPACTLY SUPPORTED CASE}

We now study self-similar behaviours that might arise for noncompactly supported data. As we will see, the situation in this case is closer to the case $\kappa_{0}=2$ that to the case $\kappa_{0}>2$, in the sense that the characteristic curves can experience during their evolution rather strong stretchings, and for this reason the class of initial data that yield self-similar solutions by means of (9)-(11) is rather large. 


\subsection{The only possible self-similar behaviour is $\kappa_{0}=2$.}

It is already implicit in the seminal paper [5] that noncompactly supported solutions of the LSW model (1)-(3) that behave asymptotically in a self-similar solution must behave necessarily like the self-similar solution (19).

Indeed, notice first that the volume conservation (12) implies

$$
\frac{d}{d \tau}\left(\int G(W, \tau) d W\right)=0
$$

Suppose that $G(W, \tau)$ solution of (14) approaches to a self-similar solution with $\kappa_{0}>2$. Then $\lambda(\tau) \rightarrow \kappa_{0}$ as $\tau \rightarrow \infty$, whence the velocity of the characteristics associated to (14) vanishes at two values $W_{1}(\tau)$, $W_{2}(\tau), 0<W_{1}(\tau)<W_{2}(\tau)<\infty$ that approach asymptotically to the two positive roots of the equation

$$
-2+3 \kappa_{0} \bar{W}_{i}^{1 / 3}-\bar{W}_{i}=0, i=1,2
$$

where $0<\bar{W}_{1}<\bar{W}_{2}<\infty$. In particular, since $\frac{\partial W\left(\tau, W_{0}\right)}{\partial W_{0}} \geq c>0$ for $W\left(\tau, W_{0}\right) \approx \bar{W}_{1}$ and $G(W, \tau)>0$ for any $W>0$, it follows analyzing the evolution of the characteristics of (14) that $G(W, \tau)$ increases exponentially in a neighbourhood of $W=\bar{W}_{1}$, and this contradicts (87).

It then follows that the only admissible self-similar behaviour for (14) is (19). Moreover, the argument above shows that, since $G$ increases exponentially along characteristics, these ones should "leak" across the critical line $W=1$ at a very precise rate in order to obtain the conservation of volume property (87). The rest of the paper is basically a description of the precise way in which this "leaking " of the characteristic curves across the critical line takes place.

\subsection{Necessary conditions for self-similar behaviour.}

Let us define

$$
\beta(\tau) \equiv \lambda(\tau)-1
$$

Since $\int_{0}^{\infty} W^{-2 / 3} \frac{G_{s}(W)}{G_{s}(0)} d W=2$, it follows from (32) that:

$$
\beta(\tau)=-\frac{\int_{0}^{\infty} W^{-2 / 3}\left(G(W, \tau)-G\left(0^{+}, \tau\right) G_{s}(W)\right) d W}{\int_{0}^{\infty} W^{-2 / 3} G(W, \tau) d W}
$$

and (14), (16) might be written as:

$$
\begin{aligned}
\frac{\partial G}{\partial \tau}+\left(-2+3 W^{1 / 3}-W+3 \beta(\tau) W^{1 / 3}\right) \frac{\partial G}{\partial W} & =G \\
G(W, 0) & =G_{0}(W)
\end{aligned}
$$

We now derive assumptions that must be made on $G_{0}(W)$ in order to have the self-similar behaviour (19) for the solutions of (89)-(91). To this end we introduce some auxiliary notation. We denote as $W\left(\tau ; W_{0}\right)$ the solutions of the characteristic equations

$$
\begin{aligned}
W_{\tau} & =-2+3 W^{1 / 3}-W+3 \beta(\tau) W^{1 / 3} \\
W\left(0 ; W_{0}\right) & =W_{0}
\end{aligned}
$$

On the other hand, we define a function $W_{0}(\tau ; W)$ by means of

$$
W\left(\tau ; W_{0}(\tau ; W)\right)=W
$$


Integrating (90) by characteristics we obtain

$$
G(W, \tau)=G_{0}\left(W_{0}(\tau ; W)\right) e^{\tau}
$$

Since $G(W, \tau)$ behaves in a self-similar manner as $\tau \rightarrow \infty$ it follows from (89) that $\beta(\tau) \rightarrow 0$ as $\tau \rightarrow \infty$. On the other hand, $W_{0}(\tau ; W) \rightarrow \infty$ as $\tau \rightarrow \infty$ uniformly on compact sets of $W \in[0,1)$. Therefore, $W\left(\tau ; W_{0}\right)$ might be approximated as $\tau \rightarrow \infty$ by means of the solutions of

$$
W_{\tau}=-2+3 W^{1 / 3}-W
$$

for $|W-1|$ of order one. On the other hand, (92) might be approximated for $|W-1|$ small to the leading order by means of

$$
W_{\tau}=-\frac{(W-1)^{2}}{3}+3 \beta(\tau)
$$

There exists a conserved quantity along the solutions of $(96)$ for $W<1$, namely

$$
F_{\text {int }}\left(W\left(\tau, W_{0}\right)\right)+\tau
$$

where

$$
F_{\text {int }}(W)=\int_{0}^{W} \frac{d \eta}{2-3 \eta^{1 / 3}+\eta}
$$

Taking into account (92), (98) as well as the fact that $\lim _{\tau \rightarrow \infty} \beta(\tau)=0$, it follows that for any $\alpha \in[0,1)$

$$
W_{0}(\tau ; W)=W_{0}\left(\tau+F_{\text {int }}(W)-F_{\text {int }}(\alpha)+\epsilon(\tau, W) ; \alpha\right)
$$

where

$$
\lim _{\tau \rightarrow \infty} \epsilon(\tau, W)=0
$$

uniformly on compact sets of $W \in[0,1)$.

Using (95), (100) as well as the fact that $G(W, \tau)$ approaches as a self-similar solution as $\tau \rightarrow \infty$ we obtain

$$
\begin{aligned}
G_{0}\left(W_{0}\left(\tau+F_{\text {int }}(W)-F_{\text {int }}(\alpha)+\epsilon(\tau, W) ; \alpha\right)\right) & =G_{0}\left(W_{0}(\tau ; W)\right)=G(W, \tau) e^{-\tau} \\
& =C e^{-\tau} e^{-F_{\text {int }}(W)}[1+o(1)]
\end{aligned}
$$

as $\tau \rightarrow \infty$, for some suitable constant $C$ that must be obtained using the volume conservation (87). In an equivalent manner, there exists a continuous, strictly increasing function $\omega(\tau)$ such that

$$
G_{0}(\omega(\tau))=C e^{F_{\text {int }}(\alpha)}[1+o(1)] e^{-\tau}
$$

as $\tau \rightarrow \infty$, where

$$
\omega(\tau)=W_{0}(\tau ; \alpha)
$$

Condition (102) provides a necessary condition that must be satisfied by the initial data $G_{0}$ in order to have self-similar behaviour for the solution of (89)-(91). Notice however that this condition is far less restrictive that the conditions (46), (66) that arise in the compactly supported case. Indeed, given an arbitrary continuous function $G_{0}(W)$ strictly decreasing we can find a continuous, strictly decreasing $\omega(\tau)$ such that (102) holds by means of:

$$
\omega(\tau)=G_{0}^{-1}\left(C e^{F_{\mathrm{int}}(\alpha)} e^{-\tau}[1+o(1)]\right)
$$

At a first glance, this suggests that any continuous, noncompactly supported, strictly decreasing initial data $G_{0}(W)$ yields self-similar behaviour as $\tau \rightarrow \infty$. Nevertheless, it turns out that this is not the case because, as it is shown in next Subsection, there exist noncompactly supported initial data $G_{0}(W)$ for 
which the long time asymptotics of the solutions of the LSW model is not self-similar. The reason for this is that an initial data, besides satisfying (102) for some function $\omega(\tau)$ must verify also that the corresponding function $\beta(\tau)$ that is uniquely defined by means of the condition (see Appendix A)

$$
W_{0}(\tau ; \alpha)=\omega(\tau)
$$

must satisfy

$$
\lim _{\tau \rightarrow \infty} \beta(\tau)=0 .
$$

since otherwise self-similar behaviour would not be possible.

\subsection{Nonselfsimilar behaviour for the LSW model.}

If $G_{0}$ is allowed to have discontinuities, it is not hard to find examples of functions $G_{0}$ where (102) fails. Indeed, let us choose a function $G_{0}$ with discontinuities at an increasing sequence of points $\left\{W_{n}: n=1,2, \ldots \infty\right\}$, satisfying $\lim _{n \rightarrow \infty} W_{n}=\infty,\left|W_{n+1}-W_{n}\right| \geq 1$, and where

$$
G_{0}\left(W_{n}^{+}\right)-G_{0}\left(W_{n}^{-}\right) \geq \frac{G_{0}\left(W_{n}^{+}\right)}{2}
$$

Condition (102) implies

$$
\lim _{\tau \rightarrow \infty} \frac{G_{0}(\omega(\tau+a))}{G_{0}(\omega(\tau))}=e^{-a}
$$

uniformly on compact sets $a \in[0, \infty)$. However, (106), (107) are incompatible. Indeed, let us define $\tau_{n}=\omega^{-1}\left(W_{n}-\frac{1}{2}\right)$. It follows from (106) that the function $\frac{G_{0}\left(\omega\left(\tau_{n}+a\right)\right)}{G_{0}\left(\omega\left(\tau_{n}\right)\right)}$ has a jump discontinuity of at least high $\frac{1}{2}$ at the value $a=\frac{1}{2}$, and this contradicts (107).

The argument above shows in a fully rigorous manner that for some discontinuous initial data $G_{0}$ the corresponding solution cannot converge to a self-similar solution as $\tau \rightarrow \infty$. The theorem below shows how to adapt the previous idea in order to obtain examples of non selfsimilar behaviour for smooth initial data $G_{0}$.

Theorem 1 There exist differentiable, strictly decreasing, noncompactly supported initial data $G_{0}(W)$ such that the corresponding solution of the problem (89)-(91) does not behave in a self-similar manner as $\tau \rightarrow \infty$.

Proof. Let us choose an increasing, but otherwise arbitrary sequence $\left\{W_{n}>0\right\}$, such that $\lim _{n \rightarrow \infty} W_{n}=$ $\infty$, as well as a sequence $\left\{\varepsilon_{n}>0\right\}$ to be precised satisfying $\lim _{n \rightarrow \infty} \varepsilon_{n}=0$. We then define a function $G_{0}(W)$ that is smooth, strictly decreasing and it has "almost discontinuities at the values $W=W_{n}$ in the sense that (see (106)):

$$
0<G_{0}\left(W_{n}+\varepsilon_{n}\right) \leq \frac{1}{2} G_{0}\left(W_{n}-\varepsilon_{n}\right)
$$

something that is certainly possible for arbitrarily small, although positive, values $\varepsilon_{n}$.

Suppose that $G(W, \tau)$, solution of (89)-(91), behaves in a self-similar manner, i.e

$$
\lim _{\tau \rightarrow \infty} G(W, \tau)=G_{s}(W)
$$

uniformly on compact sets of $W$, where $G_{s}(W)$ is as in (26) with $\kappa_{0}=2$. Moreover, without lost of generality we might assume that $C=1$. Since $G$ increases exponentially along characteristics, it then follows that the characteristic starting at $W=W_{0}$ at time $\tau=0$, arrives at a time $\tau=\bar{\tau}$ given by

$$
\bar{\tau}=\log \left(\frac{1}{G_{s}\left(W_{0}\right)}\right)+\eta\left(W_{0}\right)
$$

where $\lim _{W_{0} \rightarrow \infty} \eta\left(W_{0}\right)=0$. 
In particular, if we choose the values $\varepsilon_{n}$ sufficiently small, it follows that $G\left(W, \tau_{n}\right)$ would be "almost discontinuous" near $W=0$ for n large enough. Indeed, notice that (109) implies that $\lim _{\tau \rightarrow \infty} \beta(\tau)=0$. Then for $n$ large, the characteristic curves starting at the values $W=W_{n}-\varepsilon_{n}, W=W_{n}+\varepsilon_{n}$ decrease exponentially fast, later they remain trapped near the line $W=1$ and finally they move to the region $\{W<1-\delta\}$, with $\delta>0$ small, where they remain a time $\tau$ of order one before vanishing. At the time when the characteristic curve starting at $W=W_{n}-\varepsilon_{n}$ reaches the value $W=1+\delta$ the distance between the characteristic curves is smaller than $2 \varepsilon_{n}$. During the transition along the critical line the separation between characteristics might be estimated using Gronwall's inequality, as well as the fact that for large $\tau$, $|\beta(\tau)| \leq \frac{1}{2}$. Therefore, if $\varepsilon_{n}$ is small enough, it follows using (110) that at the time when the characteristic curve starting at $W=W_{n}-\varepsilon_{n}$ reaches $W=1-\delta$ the difference between the characteristic curves is bounded by

$$
C \varepsilon_{n} \exp \left(A \delta \log \left(\frac{1}{G_{s}\left(W_{n}-\varepsilon_{n}\right)}\right)\right)=\frac{C \varepsilon_{n}}{\left(G_{s}\left(W_{n}-\varepsilon_{n}\right)\right)^{A \delta}}
$$

where $A>0$ is a fixed constant independent on $\delta, \varepsilon_{n}$. The estimate above for the distance between characteristics is valid if $\frac{C \varepsilon_{n}}{\left(G_{s}\left(W_{n}-\varepsilon_{n}\right)\right)^{A \delta}}$ is small enough. In particular choosing the values of $\varepsilon_{n}$ satisfying

$$
\lim _{n \rightarrow \infty} \frac{\varepsilon_{n}}{\left(G_{s}\left(W_{n}-\varepsilon_{n}\right)\right)^{A \delta}}=0
$$

it follows that the characteristics starting at $W=W_{n}-\varepsilon_{n}, W=W_{n}+\varepsilon_{n}$ arrive, for $n$ large enough at distances arbitrarily small. Due to (108) it then follows that the solution of (89)-(91) cannot satisfy (109). Notice that if the values of $W_{n}$ grow fast enough, for instance satisfy $W_{n+1}-W_{n} \geq k>0$, the function $G_{s}\left(W_{0}\right)$ decays very quickly as $W_{0} \rightarrow \infty$, whence the results in [11] show that the solutions of (89)-(91) are globally defined.

\subsection{Approximating the solutions of the LSW model that are close to self- similar solutions for long times.}

\subsubsection{The transition problem.}

We now approximate asymptotically the LSW dynamics by means of a simpler problem. In a more precise manner, notice that the evolution of each characteristic curve depends, by means of the function $\beta(\tau)$ in (89) on the values of $G$ in all the characteristic curves at a given time $\tau$. On the other hand, we have seen in the previous Subsection that for solutions behaving in a self-similar manner (103) is satisfied, where $\omega(\tau)$ is given by (104). In Appendix A it is shown that the function $\beta(\tau)$ is uniquely prescribed by means of the function $W_{0}(\tau ; \alpha)$ for any fixed value of $\alpha \in[0,1)$. The term $o(1)$ in (104) does not modify the self-similar behaviour of $G(W, \tau)$. Therefore, the LSW model might be approximated by the following model:

To find $\beta(\tau)$ such that the function $W\left(\tau ; W_{0}\right)$ solution of

$$
\begin{aligned}
W_{\tau} & =-2+3 W^{1 / 3}-W+3 \beta(\tau) W^{1 / 3} \\
W\left(0 ; W_{0}\right) & =W_{0}
\end{aligned}
$$

satisfies

$$
W(\tau ; \omega(\tau))=\alpha \in[0,1)
$$

where

$$
\omega(\tau)=G_{0}^{-1}\left(C e^{F_{\text {int }}(\alpha)} e^{-\tau}\right)
$$

with

$$
C=\frac{\int_{0}^{\infty} G_{0}(W) d W}{\int_{0}^{\infty} e^{-F_{\text {int }}(W)} d W}
$$


The main advantage of the model (111)-(115), that will be denoted from now on as "transition problem", is that the integral term (89) dissappears. Moreover, problem (111)-(115) can be approximated by an explicitly solvable problem as $\tau \rightarrow \infty$.

In order to check this we need to describe the behaviour of the characteristic curves associated to (111)-(115) for long times. The characteristics arriving at $W \approx 1$ at a time $\tau>>1$ start their motion at some $W_{0}>>1$. As long as $W-1$ is of order one the solutions of (111) can be approximated by means of the solutions of (96) that are given by

$$
W\left(\tau ; W_{0}\right)=w_{\text {ext }}\left(\tau-\log \left(W_{0}\right)+F_{\text {ext }}\left(W_{0}\right)-b_{\text {ext }}\right)
$$

where:

$$
\begin{gathered}
-\log \left(w_{\text {ext }}(s)\right)+F_{\text {ext }}\left(w_{\text {ext }}(s)\right)=s+b_{\text {ext }} \\
F_{\text {ext }}(W) \equiv \int_{W}^{\infty}\left[\frac{1}{2-3 \eta^{1 / 3}+\eta}-\frac{1}{\eta}\right] d \eta=\int_{W}^{\infty} \frac{\left(3 \eta^{1 / 3}-2\right)}{\left(2-3 \eta^{1 / 3}+\eta\right) \eta} d \eta
\end{gathered}
$$

and $b_{\text {ext }}$ is defined by means of:

$$
F_{\text {ext }}(W)=\frac{3}{(W-1)}-\frac{5}{3} \log (W-1)+b_{\text {ext }}+o(1) \quad \text { as } W \rightarrow 1^{+}
$$

After reaching the region where $W$ becomes of order one the characteristics remain trapped in the region $W \approx 1$ during a very long time, due to the smallness of $\beta(\tau)$, before crossing to the region $W<1$. This stage is the crucial part of the evolution of the characteristics. In this part of the dynamics the equation for the characteristics can be approximated by means of (97). We remark that (117)-(119) yield

$$
w_{\text {ext }}(s) \sim 1+\frac{3}{s}-\frac{5 \log \left(\frac{3}{s}\right)}{s^{2}}+o\left(\frac{1}{s^{2}}\right) \quad \text { as } s \rightarrow \infty
$$

It then follows from (116) that in the "transition region" where $W \approx 1$, the characteristic $W\left(\tau ; W_{0}\right)$ matches in the region where $\left(\tau-\log \left(W_{0}\right)+F_{\text {ext }}\left(W_{0}\right)-b_{\text {ext }}\right)>>1,(W-1)^{2}>>|\beta(\tau)|$ with the unique solution of (97) that satisfies

$$
W=+\infty \quad \text { at } \quad \tau=\log \left(W_{0}\right)-F_{\text {ext }}\left(W_{0}\right)+b_{\text {ext }}
$$

On the other hand, let us suppose that the characteristic curve $W\left(\tau ; W_{0}\right)$ satisfies $W\left(\bar{\tau} ; W_{0}\right)=\bar{W}$. Using the approximation (96) in the region where $(1-W)$ is of order one it would follow that

$$
W\left(\tau ; W_{0}\right)=w_{\text {int }}\left(\tau-\bar{\tau}-F_{\text {int }}(\bar{W})+b_{\text {int }}\right)
$$

where

$$
\begin{gathered}
F_{\text {int }}\left(w_{\text {int }}(s)\right)=b_{\text {int }}-s \\
F_{\text {int }}(W) \sim \frac{3}{(1-W)}+\frac{5}{3} \log (1-W)+b_{\text {int }}+o(1) \text { as } W \rightarrow 1^{-}
\end{gathered}
$$

and where $F_{\text {int }}$ is as in (99). Formulae (123), (124) yield:

$$
w_{\text {int }}(s) \sim 1+\frac{3}{s}-\frac{5 \log \left(-\frac{3}{s}\right)}{s^{2}}+o\left(\frac{1}{s^{2}}\right) \text { as } s \rightarrow-\infty
$$

Therefore (122) and (125) imply that $W\left(\tau ; W_{0}\right)$ matches for $\left(\tau-\bar{\tau}-F_{\text {int }}(\bar{W})+b_{\text {int }}\right)>>1,(W-1)^{2}>>$ $|\beta(\tau)|$ with the unique solution of $(97)$ satisfying

$$
W=-\infty \text { at } \tau=\bar{\tau}+F_{\text {int }}(\bar{W})-b_{\text {int }}
$$


At a first glance there seems to be something contradictory in the fact that $|W|$ becomes unbounded near $\tau=\log \left(W_{0}\right)-F_{\text {ext }}\left(W_{0}\right)+b_{\text {ext }}$ and $\tau=\bar{\tau}+F_{\text {int }}(\bar{W})-b_{\text {int }}$. Notice, however that the function $W$ is approximated by means of the solution of (97) satisfying (121) only in those regions where $|W-1|<<1$.

Let us define $X=W-1$. Combining (97), (121), it follows that $X$ satisfies for each $\bar{\tau}$

$$
\begin{aligned}
X_{\tau} & =-\frac{X^{2}}{3}+3 \beta(\tau) \quad, \quad S(\bar{\tau})<\tau<\bar{\tau} \\
X(S(\bar{\tau})) & =+\infty, X(\bar{\tau})=-\infty
\end{aligned}
$$

where the function $S(\bar{\tau})$ has been defined by means of the equation

$$
S\left(\bar{\tau}+F_{\text {int }}(\bar{W})-b_{\text {int }}\right)=\log \left(W_{0}\right)-F_{\text {ext }}\left(W_{0}\right)+b_{\text {ext }}
$$

In particular, choosing $\bar{W}=\alpha$, and using (113) we obtain that

$$
S\left(\bar{\tau}+F_{\text {int }}\left(\frac{1}{2}\right)-b_{\text {int }}\right)=\log (\omega(\bar{\tau}))-F_{\text {ext }}(\omega(\bar{\tau}))+b_{\text {ext }}
$$

where $\omega(\bar{\tau})$ is as in (114), whence

$$
S(\tau)=\log \left(\omega\left(\tau-F_{\mathrm{int}}\left(\frac{1}{2}\right)+b_{\mathrm{int}}\right)\right)-F_{\mathrm{ext}}\left(\omega\left(\tau-F_{\mathrm{int}}\left(\frac{1}{2}\right)+b_{\mathrm{int}}\right)\right)+b_{\mathrm{ext}}
$$

Therefore, as $\tau \rightarrow \infty$, the problem (111)-(115) might be approximated by means of (114), (127), (128), (131). This "approximated transition problem" is the main ingredient in order to study the asymptotics of both the transition problem (111)-(115) and the whole LSW model as $\tau \rightarrow \infty$.

\subsubsection{On the explicit solution of the approximate transition problem.}

Tthe approximate transition problem (114), (127), (128), (131) can be explicitly solved. We will assume by convenience that the function $S(\tau)$ defined by means of (131) has three derivatives, something that due to (114) equivales to assuming that $G_{0}$ is differentiable enough.

We rewrite here the approximate transition problem by convenience. The problem is, given a strictly monotonic increasing function $S(\cdot)$ satisfying $S(x)<x$, for $x>0$, to find functions $\bar{\beta}(\tau)$ such that for any $\bar{\tau}>S^{-1}\left(T_{0}\right)$ :

$$
\begin{aligned}
X_{\tau} & =-\frac{1}{3} X^{2}+3 \beta(\tau), \quad S(\bar{\tau})<\tau<\bar{\tau} \\
X\left((S(\bar{\tau}))^{+}\right) & =+\infty, X\left((\bar{\tau})^{-}\right)=-\infty
\end{aligned}
$$

(i) A particular solution of the approximate transition problem.

There are several different ways of solving the problem (132), (133). The method used in this Section is convenient in order to treat in a perturbative manner the whole transition problem (111)-(115).

Let us define a function $f(\zeta) \geq T_{0}$ strictly monotonically increasing in $\zeta$, defined in $\zeta \geq \zeta_{0}$ satisfying:

$$
\begin{aligned}
S(f(\zeta+1)) & =f(\zeta) \\
f\left(\zeta_{0}\right) & =T_{0}
\end{aligned}
$$

where $S(\cdot)$ is the function in (133).

Our assumptions imply the existence of the inverse function $S^{-1}(\cdot)$. Therefore, given an arbitrary function $f(\zeta)$ in $\left[\zeta_{0}, \zeta_{0}+1\right)$ satisfying (135) we obtain $f$ defined in $\left[\zeta_{0}, \infty\right)$ iterating the formula:

$$
f(\zeta+1)=S^{-1}(f(\zeta)) .
$$


In further computations we will need $f$ to be differentiable three times. The function $f$ defined by means of (136) has this regularity if the function $f(\zeta)$ defined in $\left[\zeta_{0}, \zeta_{0}+1\right)$ that will be assumed to be monotonically increasing in this interval belongs to $C^{3}\left[\zeta_{0}, \zeta_{0}+1\right]$ and satisfies the following compatibility conditions:

$$
\begin{aligned}
f\left(\zeta_{0}\right) & =f\left(\zeta_{0}+1\right) \\
f^{\prime}\left(\zeta_{0}\right) & =S^{\prime}\left(f\left(\zeta_{0}+1\right)\right) f^{\prime}\left(\zeta_{0}+1\right) \\
f^{\prime \prime}\left(\zeta_{0}\right) & =S^{\prime \prime}\left(f\left(\zeta_{0}+1\right)\right)\left(f^{\prime}\left(\zeta_{0}+1\right)\right)^{2}+S^{\prime}\left(f\left(\zeta_{0}+1\right)\right) f^{\prime \prime}\left(\zeta_{0}+1\right) \\
f^{\prime \prime \prime}\left(\zeta_{0}\right) & =S^{\prime \prime \prime}\left(f\left(\zeta_{0}+1\right)\right)\left(f^{\prime}\left(\zeta_{0}+1\right)\right)^{3}+ \\
& 3 S^{\prime \prime}\left(f\left(\zeta_{0}+1\right)\right) f^{\prime}\left(\zeta_{0}+1\right) f^{\prime \prime}\left(\zeta_{0}+1\right)+S^{\prime}\left(f\left(\zeta_{0}+1\right)\right) f^{\prime \prime \prime}\left(\zeta_{0}+1\right)
\end{aligned}
$$

Under these assumptions the monotonically increasing function $f$ defined by means of (136) belongs to $C^{3}\left[\zeta_{0}, \infty\right)$ and satisfies:

$$
\lim _{\zeta \rightarrow \infty} f(\zeta)=\infty
$$

Given such a function $f(\cdot)$ we define a new function $\chi(Y, \zeta)$ as:

$$
\chi(Y, \zeta)=\frac{3 Y}{f^{\prime}(\zeta)}+\frac{3 f^{\prime \prime}(\zeta)}{2\left(f^{\prime}(\zeta)\right)^{2}}
$$

Let us introduce the change of variables:

$$
\begin{aligned}
\tau & =f(\zeta) \\
X & =\chi(Y, \zeta)
\end{aligned}
$$

We can then obtain a solution of $(132),(133) \beta(\tau)=\bar{\beta}(\tau)$, given by:

$$
\bar{\beta}(f(\zeta))=\frac{1}{\left(f^{\prime}(\zeta)\right)^{2}}\left(\frac{1}{2}\{f, \zeta\}-\pi^{2}\right)
$$

where $\{f, \zeta\}$ is the Schwartzian derivative (cf. for instance [4]):

$$
\{f, \zeta\} \equiv \frac{f^{\prime \prime \prime}(\zeta)}{f^{\prime}(\zeta)}-\frac{3}{2}\left(\frac{f^{\prime \prime}(\zeta)}{f^{\prime}(\zeta)}\right)^{2}
$$

Under this assumption the change of variables (142), (143) transforms the original transition problem (132)-(133) into:

$$
\begin{aligned}
\frac{d Y}{d \zeta}+Y^{2}+\pi^{2} & =0, \zeta>\zeta_{\mathrm{arr}} \\
Y\left((\bar{\zeta})^{+}\right) & =+\infty \\
Y\left((\bar{\zeta}+1)^{-}\right) & =-\infty
\end{aligned}
$$

where $\bar{\tau}=f(\bar{\zeta})$, and we have used (133), (134) to obtain (148).

The problem (146)-(148) can be easily solved, since the solution of (146), (147) is given by:

$$
Y=Y\left(\zeta, \zeta_{0}\right)=\pi \operatorname{coth}(\pi(\zeta-\bar{\zeta}))
$$

and it can be inmediately checked that $Y\left(\zeta, \zeta_{0}\right)$ satisfies (148).

Let us summarize. Given $f \in C^{3}\left[\zeta_{0}, \zeta_{0}+1\right]$ satisfying the compatibility conditions (137)-(140) we can obtain $f \in C^{3}\left[\zeta_{0}, \infty\right)$ by means of (136). Then, the function $\beta(\tau)$ defined in (144) provides a solution of (132)-(133). 
Let us mention a particular class of functions $S$ that we will use repeatedly in the following. Suppose that the initial data $G_{0}$ satisfies:

$$
\frac{G_{0}\left(w_{e x t}\left(-X_{0}-\lambda\left(X_{0}\right) \zeta\right)\right)}{G_{0}\left(w_{e x t}\left(-X_{0}\right)\right)}=e^{-\zeta\left(1+\delta\left(X_{0}, \zeta\right)\right)} \text { as } X_{0} \rightarrow \infty
$$

where $w_{e x t}$ is as in (117), where $\delta\left(X_{0}, \zeta\right) \rightarrow 0$ as $X_{0} \rightarrow \infty$ uniformly on compact set of $\zeta$, and where

$$
\lambda\left(X_{0}\right) \sim \frac{C}{\left(X_{0}\right)^{\alpha}} \text { as } W_{0} \rightarrow \infty
$$

for some $0<\alpha<1$. Examples of initial data satisfying (150), (151) are, for instance:

$$
G_{0}(W) \sim C W^{B}(\log (W))^{D} e^{-W^{A}} \text { as } W \rightarrow \infty, A>0, B, D \in \mathbb{R}
$$

for which (150) holds with

$$
\lambda\left(X_{0}\right) \sim \frac{1}{A} \frac{1}{\left(X_{0}\right)^{A}} \quad \text { as } W \rightarrow \infty
$$

We can compute the asymptotics of the function $S(\cdot)$ for initial data with the form (150), (151) as follows. Using (114) we obtain:

$$
G_{0}\left(w_{\text {ext }}\left(-X_{0}\right)\right)=C e^{F_{\mathrm{int}}(\alpha)} e^{-\omega^{-1}\left(w_{e x t}\left(-X_{0}\right)\right)}
$$

Then

$$
\frac{G_{0}\left(w_{\text {ext }}\left(-X_{0}-\lambda\left(X_{0}\right) \zeta\right)\right)}{G_{0}\left(w_{\text {ext }}\left(-X_{0}\right)\right)}=e^{-\left[\omega^{-1}\left(w_{\text {ext }}\left(-X_{0}-\lambda\left(X_{0}\right) \zeta\right)\right)-\omega^{-1}\left(w_{\text {ext }}\left(-X_{0}\right)\right)\right]}
$$

It then follows from (150) that

$$
\lim _{X_{0} \rightarrow \infty} \frac{\omega^{-1}\left(w_{\text {ext }}\left(-X_{0}-\lambda\left(X_{0}\right) \zeta\right)\right)-\omega^{-1}\left(w_{\text {ext }}\left(-X_{0}\right)\right)}{\zeta}=1
$$

for any $\zeta>0$, and since $w_{\text {ext }}\left(-X_{0}\right) \sim C e^{X_{0}}$ as $X_{0} \rightarrow \infty$, for some $C>0$, this formula equivales to

$$
\lim _{X_{0} \rightarrow \infty} \frac{\omega^{-1}\left(w_{\text {ext }}\left(-X_{0}\right)\left(1+\lambda\left(X_{0}\right) \zeta\right)\right)-\omega^{-1}\left(w_{\text {ext }}\left(-X_{0}\right)\right)}{\zeta}=1
$$

It turns out, using (151) that the solution of this equation yields the asymptotic formula

$$
\omega^{-1}(Y) \sim K Y^{1-\alpha} \text { as } Y \rightarrow \infty
$$

for some $K>0$, as it can be checked by means of a direct computation. It then follows from (131) that

$$
S(\tau) \sim a \log (\tau) \text { as } \tau \rightarrow \infty
$$

where $a=\frac{1}{1-\alpha}$.

(ii) On the general solution of the approximate transition problem.

We can study the general solution of (132)-(133) as follows. Let us denote as $\bar{\beta}(\tau)$ an arbitrary solution of (132)-(133) given by (144) for some function $f(\zeta)$ fixed. Using the change of variables (142), (143) and introducing a new function $Z$ by means of

$$
Y=\pi \cot (\pi(\zeta-\bar{\zeta}-Z))
$$

we transform (132)-(133) into

$$
\begin{aligned}
Z_{\zeta} & =\frac{\sin ^{2}(\pi(\zeta-\bar{\zeta}-Z))}{\pi^{2}} \lambda(\zeta) \\
Z(\zeta ; \bar{\zeta}) & =Z(\zeta ; \bar{\zeta}+1)=0
\end{aligned}
$$


where

$$
\lambda(\zeta)=(\beta(\tau)-\bar{\beta}(\tau))\left(f^{\prime}(\zeta)\right)^{2}
$$

Integrating (155) in the interval $(\bar{\zeta}, \bar{\zeta}+1)$ and using (156) we obtain

$$
\int_{\bar{\zeta}}^{\bar{\zeta}+1} \sin ^{2}(\pi(\zeta-\bar{\zeta}-Z(\zeta ; \bar{\zeta}))) \lambda(\zeta) d \zeta=0
$$

Differentiating (158) we arrive at:

$$
\int_{\bar{\zeta}}^{\bar{\zeta}+1} \sin (2 \pi(\zeta-\bar{\zeta}-Z(\zeta, \bar{\zeta})))\left(1+\frac{\partial Z(\zeta, \bar{\zeta})}{\partial \bar{\zeta}}\right) \lambda(\zeta) d \zeta=0
$$

We compute $\frac{\partial Z(\zeta, \bar{\zeta})}{\partial \bar{\zeta}}$ differentiating (155), (156) with respect to $\bar{\zeta}$. Then:

$$
\begin{aligned}
\left(\frac{\partial Z}{\partial \bar{\zeta}}\right)_{\zeta} & =-\frac{\sin (2 \pi(\zeta-\bar{\zeta}-Z(\zeta, \bar{\zeta}))) \lambda(\zeta)}{\pi}\left(1+\frac{\partial Z}{\partial \bar{\zeta}}\right) \\
\frac{\partial Z}{\partial \bar{\zeta}}(\bar{\zeta}, \bar{\zeta}) & =\frac{\partial Z}{\partial \bar{\zeta}}(\bar{\zeta}+1, \bar{\zeta})=0
\end{aligned}
$$

Solving (160), (161) we obtain:

$$
\frac{\partial Z}{\partial \bar{\zeta}}(\zeta, \bar{\zeta})=-\int_{\bar{\zeta}}^{\zeta} e^{-\bar{\psi}(\zeta, \bar{\zeta})+\bar{\psi}(\eta, \bar{\zeta})} \frac{\partial \psi(\eta, \bar{\zeta})}{\partial \zeta} d \eta=\left(e^{-\bar{\psi}(\zeta, \bar{\zeta})}-1\right)
$$

where

$$
\psi(\zeta, \bar{\zeta}) \equiv \frac{1}{\pi} \int_{\bar{\zeta}}^{\zeta} \sin (2 \pi(\eta-\bar{\zeta}-Z(\eta, \bar{\zeta}))) \lambda(\eta) d \eta
$$

Using (162) in (159) we deduce that:

$$
0=\int_{\bar{\zeta}}^{\bar{\zeta}+1} \frac{\partial \psi(\zeta, \bar{\zeta})}{\partial \zeta} e^{-\psi(\zeta, \bar{\zeta})} d \zeta=e^{-\psi(\bar{\zeta}+1, \bar{\zeta})}-1
$$

or, equivalently

$$
\psi(\bar{\zeta}+1, \bar{\zeta})=\frac{1}{\pi} \int_{\bar{\zeta}}^{\bar{\zeta}+1} \sin (2 \pi(\zeta-\bar{\zeta}-Z(\zeta, \bar{\zeta}))) \lambda(\zeta) d \zeta=0
$$

Differentiating (164) with respect to $\bar{\zeta}$ and using again (162) we arrive at

$$
\int_{\bar{\zeta}}^{\bar{\zeta}+1} \cos (2 \pi(\zeta-\bar{\zeta}-Z(\zeta, \bar{\zeta}))) e^{-\psi(\zeta, \bar{\zeta})} \lambda(\zeta) d \zeta=0
$$

Differentiating once more (165) with respect to $\bar{\zeta}$ and using (162), (164) we obtain

$$
\lambda(\bar{\zeta}+1)-\lambda(\bar{\zeta})=\int_{\bar{\zeta}}^{\bar{\zeta}+1} K(\eta, \bar{\zeta}) \lambda(\eta) d \eta
$$

where

$$
\begin{aligned}
K(\eta, \bar{\zeta}) & \equiv\left[2 \pi \sin (2 \pi(\eta-\bar{\zeta}-Z(\eta, \bar{\zeta}))) e^{-2 \psi(\eta, \bar{\zeta})}\right. \\
& \left.+\cos (2 \pi(\eta-\bar{\zeta}-Z(\eta, \bar{\zeta}))) e^{-\psi(\eta, \bar{\zeta})} \frac{\partial \psi(\eta, \bar{\zeta})}{\partial \bar{\zeta}}\right]
\end{aligned}
$$


Using (163) it then follows that:

$$
\begin{aligned}
\int_{\bar{\zeta}}^{\bar{\zeta}+1} K(\eta, \bar{\zeta}) \lambda(\eta) d \eta & =\int_{\bar{\zeta}}^{\bar{\zeta}+1}\left[2 \pi \sin (2 \pi(\eta-\bar{\zeta}-Z(\eta, \bar{\zeta}))) e^{-2 \psi(\eta, \bar{\zeta})}\right. \\
& \left.+\cos (2 \pi(\eta-\bar{\zeta}-Z(\eta, \bar{\zeta}))) e^{-\psi(\eta, \bar{\zeta})} \frac{\partial \psi(\eta, \bar{\zeta})}{\partial \bar{\zeta}}\right] \lambda(\eta) d \eta \\
& =2 \pi^{2} \int_{\bar{\zeta}}^{\bar{\zeta}+1} \frac{\partial \psi(\eta, \bar{\zeta})}{\partial \zeta} e^{-2 \psi(\eta, \bar{\zeta})} d \eta \\
& -\frac{1}{\pi} \int_{\bar{\zeta}}^{\bar{\zeta}+1} d \eta \lambda(\eta) \cos (2 \pi(\eta-\bar{\zeta}-Z(\eta, \bar{\zeta}))) e^{-\psi(\eta, \bar{\zeta})} \\
& \int_{\bar{\zeta}}^{\eta} \cos (2 \pi(\xi-\bar{\zeta}-Z(\xi, \bar{\zeta})))\left(1+\frac{\partial Z}{\partial \bar{\zeta}}(\xi, \bar{\zeta})\right) \lambda(\xi) d \xi
\end{aligned}
$$

whence, using (162), (164), (165) we can then write:

$$
\begin{aligned}
& \int_{\bar{\zeta}}^{\bar{\zeta}+1} K(\eta, \bar{\zeta}) \lambda(\eta) d \eta \\
& =\pi^{2}\left(1-e^{-2 \psi(\bar{\zeta}+1, \bar{\zeta})}\right)-\frac{1}{2 \pi}\left(\int_{\bar{\zeta}}^{\bar{\zeta}+1} \cos (2 \pi(\eta-\bar{\zeta}-Z(\eta, \bar{\zeta}))) e^{-\psi(\eta, \bar{\zeta})} \lambda(\eta) d \eta\right)^{2}=0
\end{aligned}
$$

Plugging this formula into (166) we finally arrive at

$$
\lambda(\bar{\zeta}+1)-\lambda(\bar{\zeta})=0
$$

Let us summarize: Given $S \in C^{3}\left[\zeta_{0}, \infty\right)$ we define $\bar{\beta}(\tau)$ by means of (144). Then, any smooth function $\beta(\tau)$ solving (132), (133) can be written as in (157), where $\lambda(\zeta)$ satisfies the periodicity condition (167).

In particular, this representation formula for the solutions of (132), (133) shows that this problem has a very large degree of nonuniqueness. Moreover, formulae (157), (167) shows that two solutions $\beta(\tau)$ of $(132),(133)$ differ in a function $\frac{\lambda(\zeta)}{\left(f^{\prime}(\zeta)\right)^{2}}$. Actually this difference of terms is very small compared with the leading order asymptotics of the function $\beta(\tau)$ as $\tau \rightarrow \infty$. More precisely, for functions $S$ satisfying $(153)$ the term $\frac{\lambda(\zeta)}{\left(f^{\prime}(\zeta)\right)^{2}}$ is basically the correction "beyond all the orders" whose analysis plays a crucial role in [15]. The analysis in this Subsection avoids the use of explicit asymptotics for the function $S$ and provides a much simpler description of the transition of the characteristic curves across the critical line that the one in that paper.

In the rest of this paper we approximate the dynamics of both the transition problem (111)-(115) and the whole LSW dynamics as perturbations of the equation (167). The main result of this paper is the possibility of describing the LSW dynamics as a perturbation of the explicitly solvable problem studied in this Subsection.

\section{(iii) On the asymptotics of the function $\beta(\tau)$.}

It is interesting to verify that the function $\beta(\tau)$ defined by means of (144), (157) has the asymptotic behaviours computed in [5], [15] for functions $S$ satisfying (153). Indeed, differentiating (134) three times and using (153) we obtain the approximations:

$$
\begin{aligned}
& a f^{\prime}(\zeta+1)=f(\zeta+1) f^{\prime}(\zeta) \\
& \frac{f^{\prime \prime}(\zeta+1)}{f^{\prime}(\zeta+1)}=\frac{f^{\prime \prime}(\zeta)}{f^{\prime}(\zeta)}+\frac{1}{a} f^{\prime}(\zeta)
\end{aligned}
$$




$$
\frac{f^{\prime \prime \prime}(\zeta+1)}{f^{\prime}(\zeta+1)}=\frac{f^{\prime \prime \prime}(\zeta)}{f^{\prime}(\zeta)}+\frac{3}{a} f^{\prime \prime}(\zeta)+\frac{1}{a^{2}}\left(f^{\prime}(\zeta)\right)^{2}
$$

Using (169), (170) in (145) we obtain the following transformation law for the Schwartzian derivative:

$$
\{f, \zeta\}=-\frac{1}{2 a^{2}}\left(f^{\prime}(\zeta-1)\right)^{2}+\{f, \zeta-1\}
$$

and plugging this formula into (144) we obtain, using also (168):

$$
\bar{\beta}(f(\zeta))=-\frac{1}{4} \frac{1}{(f(\zeta))^{2}}+\left(\frac{f^{\prime}(\zeta-1)}{f^{\prime}(\zeta)}\right)^{2} \bar{\beta}(f(\zeta-1))
$$

whence, using (134), (142), (168):

$$
\bar{\beta}(\tau)=-\frac{1}{4} \frac{1}{(\tau)^{2}}+\left(\frac{a}{\tau}\right)^{2} \bar{\beta}(S(\tau))
$$

Iterating this formula we obtain the asymptotics:

$$
\bar{\beta}(\tau) \sim-\frac{1}{4}\left[\frac{1}{(\tau)^{2}}+\frac{1}{(\tau)^{2}(\log (\tau))^{2}}+\frac{1}{(\tau)^{2}(\log (\tau))^{2}(\log (\log (\tau)))^{2}}+\ldots\right]
$$

as $\tau \rightarrow \infty$. This asymptotics has been obtained in [5], [15] using different methods. Let us remark that the difference between the functions $\beta(\tau)$ and $\bar{\beta}(\tau)$ differs is a term smaller than all the terms in the series on the right hand side of (172). Indeed, due to (167) it follows that $\lambda$ is bounded. Therefore, (157) implies that the difference $\beta(\tau)-\bar{\beta}(\tau)$ is of order $\frac{1}{\left(f^{\prime}(\zeta)\right)^{2}}$. Due to (168) we have that this term must be understood as

$$
\frac{1}{\left(f^{\prime}(\zeta)\right)^{2}} \sim \frac{1}{(\tau \log (\tau) \log \log (\tau) \ldots .)^{2}}
$$

as $\tau \rightarrow \infty$, whence it is smaller than all the terms in (172). Corrective terms analogous to (173) have been computed in [15] using more involved, explicit computations.

\subsubsection{Long time asymptotics for the transition problem.}

In this Subsection we study the long time asymptotics for the transition problem (111)-(115) that we recall here by convenience. The problem is: To find $\tilde{\beta}(\tau)$ such that the solution of

$$
\begin{aligned}
W_{\tau} & =-2+3 W^{1 / 3}-W+3 \tilde{\beta}(\tau) W^{1 / 3} \\
W\left(\tau_{0} ; W_{0}\right) & =W_{0}
\end{aligned}
$$

satisfies:

$$
W(\tau ; \omega(\tau))=\alpha
$$

where $\omega(\tau)$ is as in (114). We will assume from now on that $\omega(\tau)$ is as differentiable as required by the computations.

Several of the estimates formally derived in this and in the next Subsection have been rigorously proved in [17], where some analogous problems have been studied using similar ideas.

Our goal is to approximate this problem by means of the approximate transition problem (132), (133). Let us denote as $\bar{\beta}(\tau)$ a solution of this problem given by (142), (144).

To study the long time asymptotics of the solutions of (174)-(176) we use a change of variables similar to (142), (143), (154), namely:

$$
\begin{aligned}
\tau & =f(\zeta) \\
W-1 & =\frac{3 Y}{f^{\prime}(\zeta)}+\frac{3 f^{\prime \prime}(\zeta)}{2\left(f^{\prime}(\zeta)\right)^{2}} \\
Y & =\pi \cot (\pi(\zeta-\bar{\zeta}-Z))
\end{aligned}
$$


where $Z=Z\left(\zeta, \bar{\zeta}, \zeta_{0}\right)$ and $\bar{\tau}=f(\bar{\zeta}+1), \tau_{0}=f\left(\zeta_{0}\right)$. These changes transform (174)-(176) into:

$$
\begin{aligned}
Z_{\zeta} & =\frac{\sin ^{2}(\pi(\zeta-\bar{\zeta}-Z))}{\pi^{2}}\left[\lambda(\zeta)+R\left(Z, \zeta, \bar{\zeta}, \zeta_{0}\right)\right] \\
Z & =\left(\zeta_{0}-\zeta\right)-\frac{1}{\pi} \operatorname{arccot}\left(\frac{f^{\prime}\left(\zeta_{0}\right)}{3 \pi}\left(\bar{W}_{0}(\bar{\tau}, \alpha)-1-\frac{3 f^{\prime \prime}\left(\zeta_{0}\right)}{2\left(f^{\prime}\left(\zeta_{0}\right)\right)^{2}}\right)\right), \zeta=\zeta_{0} \\
Z & =\frac{1}{\pi} \operatorname{arccot}\left(\frac{f^{\prime}(\bar{\zeta}+1)}{3 \pi}\left[(1-\alpha)+\frac{3 f^{\prime \prime}(\bar{\zeta}+1)}{2\left(f^{\prime}(\bar{\zeta}+1)\right)^{2}}\right]\right), \zeta=\bar{\zeta}+1
\end{aligned}
$$

where:

$$
\begin{aligned}
\mu(\zeta) & =\tilde{\beta}(\tau)-\bar{\beta}(\tau) \\
\lambda(\zeta) & =\mu(\zeta)\left(f^{\prime}(\zeta)\right)^{2} \\
R\left(Z, \zeta, \bar{\zeta}, \zeta_{0}\right) & =\left(f^{\prime}(\zeta)\right)^{2}\left[\frac{h_{1}(W)}{3}+\beta(f(\zeta)) h_{2}(W)\right] \\
h_{1}(W) & =\frac{(W-1)^{2}}{3}-2+3 W^{1 / 3}-W \\
h_{2}(W) & =W^{1 / 3}-1
\end{aligned}
$$

The problem (180)-(182) might be considered as a perturbation of the exactly solvable problem (155), (156). It turns out, however, that the presence of the boundary conditions (181), (182) instead of (156) force to introduce some "boundary effects" that we pass to discuss in detail.

Many of the computations below are valid for rather general functions $S(\cdot)$. Nevertheless, we will assume by definiteness that $S$ satisfies. (153). This includes in particular initial data like the ones in (152). Under this assumption we can think on the function $f(\zeta)$ in (142) as

$$
f(\zeta) \approx \exp \left(\frac{1}{a} \exp \left(\ldots\left(\frac{1}{a} \exp \left(\frac{\zeta}{a}\right)\right)\right)\right) \quad \text { as } \zeta \rightarrow \infty
$$

Several properties of the function $f$ and its derivatives under the assumption (153) are collected in Appendix B.

Integrating (180) and using the boundary conditions (181), (182) we obtain:

$$
\begin{aligned}
& \int_{\zeta_{0}}^{\bar{\zeta}+1} \frac{\sin ^{2}\left(\pi\left(\zeta-\bar{\zeta}-Z\left(\zeta, \bar{\zeta}, \zeta_{0}\right)\right)\right)}{\pi^{2}}\left[\lambda(\zeta)+R\left(Z\left(\zeta, \bar{\zeta}, \zeta_{0}\right), \zeta, \bar{\zeta}, \zeta_{0}\right)\right] d \zeta \\
& =\theta\left(\bar{\zeta}, \zeta_{0}\right)-\left(\zeta_{0}-\bar{\zeta}\right)
\end{aligned}
$$

where

$$
\begin{gathered}
\theta\left(\bar{\zeta}, \zeta_{0}\right) \equiv \frac{1}{\pi} \operatorname{arccot}\left(\frac{f^{\prime}(\bar{\zeta}+1)}{3 \pi}\left[(1-\alpha)+\frac{3 f^{\prime \prime}(\bar{\zeta}+1)}{2\left(f^{\prime}(\bar{\zeta}+1)\right)^{2}}\right]\right)+ \\
\frac{1}{\pi} \operatorname{arccot}\left(\frac{f^{\prime}\left(\zeta_{0}\right)}{3 \pi}\left(\bar{W}_{0}(\bar{\tau}, \alpha)-1-\frac{3 f^{\prime \prime}\left(\zeta_{0}\right)}{2\left(f^{\prime}\left(\zeta_{0}\right)\right)^{2}}\right)\right)
\end{gathered}
$$

Equation (188) is reminiscent of (158). Actually, (188) can be transformed to a form closer to (158) by means of some algebraic manipulations. Using the trigonometric identity $\sin ^{2}(x)=\frac{1}{1+\cot ^{2}(x)}$ as well as (179) we obtain:

$$
\frac{\sin ^{2}\left(\pi\left(\zeta-\bar{\zeta}-Z\left(\zeta, \bar{\zeta}, \zeta_{0}\right)\right)\right)}{\pi^{2}}=\frac{9}{9 \pi^{2}+\left(f^{\prime}(\zeta)\right)^{2}\left(W-1-\frac{3 f^{\prime \prime}(\zeta)}{2\left(f^{\prime}(\zeta)\right)^{2}}\right)^{2}}
$$


Therefore, the second term on the left of (188) becomes:

$$
\begin{aligned}
& \int_{\zeta_{0}}^{\bar{\zeta}+1} \frac{\sin ^{2}\left(\pi\left(\zeta-\bar{\zeta}-Z\left(\zeta, \bar{\zeta}, \zeta_{0}\right)\right)\right)}{\pi^{2}} R\left(Z\left(\zeta, \bar{\zeta}, \zeta_{0}\right), \zeta, \bar{\zeta}, \zeta_{0}\right) d \zeta-\left(\bar{\zeta}-\zeta_{0}\right) \\
& =\int_{\zeta_{0}}^{\bar{\zeta}+1}\left[\frac{3\left(f^{\prime}(\zeta)\right)^{2} h_{1}(W)}{9 \pi^{2}+\left(f^{\prime}(\zeta)\right)^{2}\left(W-1-\frac{3 f^{\prime \prime}(\zeta)}{2\left(f^{\prime}(\zeta)\right)^{2}}\right)^{2}}-\chi(\bar{\zeta}-\zeta)\right] d \zeta \\
& +\int_{\zeta_{0}}^{\bar{\zeta}+1} \frac{9\left(f^{\prime}(\zeta)\right)^{2} \beta(f(\zeta)) h_{2}(W)}{9 \pi^{2}+\left(f^{\prime}(\zeta)\right)^{2}\left(W-1-\frac{3 f^{\prime \prime}(\zeta)}{2\left(f^{\prime}(\zeta)\right)^{2}}\right)^{2}} d \zeta \\
& \equiv I_{1}+I_{2}
\end{aligned}
$$

where $\chi(s)=1, s \geq 0, \chi(s)=0, s<0$, and $h_{1}(W), h_{2}(W)$ are as in (186), (187).

Since $\beta(\tau) \rightarrow 0$ we can approximate the function $W$ in the integrals $I_{1}, I_{2}$ as

$$
W \sim w_{\text {ext }}(f(\zeta)-f(\bar{\zeta}))
$$

for $\zeta_{0} \leq \zeta \leq \bar{\zeta}+\delta_{1}(\bar{\zeta})$, where $\delta_{1}(\bar{\zeta}) \sim \frac{1}{\left(f^{\prime}(\bar{\zeta})\right)^{\alpha}}$, and

$$
W \sim w_{\text {int }}\left(f(\zeta)-f(\bar{\zeta}+1)-F_{\text {int }}(\alpha)\right)
$$

for $(\bar{\zeta}+1)-\delta_{2}(\bar{\zeta}) \leq \zeta \leq \bar{\zeta}+1$, where $\delta_{2}(\bar{\zeta}) \sim \frac{1}{\left(f^{\prime}(\bar{\zeta}+1)\right)^{\alpha}}, 0<\alpha<1$. Due to the fast growth of the function $f(\zeta)$ as $\zeta \rightarrow \infty$, it turns out that the main contribution to the integrals $I_{1}, I_{2}$ is due to the intervals $\left[\zeta_{0}, \bar{\zeta}+\delta_{1}(\bar{\zeta})\right]$. Then:

$$
\begin{aligned}
& I_{1} \sim \int_{\zeta_{0}}^{\bar{\zeta}+\delta_{1}(\bar{\zeta})} \Phi_{1}(f(\zeta)-f(\bar{\zeta})) d \zeta \\
& I_{2} \sim \int_{\zeta_{0}}^{\bar{\zeta}+\delta_{1}(\bar{\zeta})} \Phi_{2}(f(\zeta)-f(\bar{\zeta})) d \zeta
\end{aligned}
$$

where

$$
\begin{aligned}
\Phi_{1}(f(\zeta)-f(\bar{\zeta})) & \equiv\left[\frac{3\left(f^{\prime}(\zeta)\right)^{2} h_{1}\left(w_{\text {ext }}(f(\zeta)-f(\bar{\zeta}))\right)}{9 \pi^{2}+\left(f^{\prime}(\zeta)\right)^{2}\left(w_{\text {ext }}(f(\zeta)-f(\bar{\zeta}))-1-\frac{3 f^{\prime \prime}(\zeta)}{2\left(f^{\prime}(\zeta)\right)^{2}}\right)^{2}}-\chi(\bar{\zeta}-\zeta)\right] \\
\Phi_{2}(f(\zeta)-f(\bar{\zeta})) & \equiv \frac{9\left(f^{\prime}(\zeta)\right)^{2} \beta(f(\zeta)) h_{2}\left(w_{\text {ext }}(f(\zeta)-f(\bar{\zeta}))\right)}{9 \pi^{2}+\left(f^{\prime}(\zeta)\right)^{2}\left(w_{\text {ext }}(f(\zeta)-f(\bar{\zeta}))-1-\frac{3 f^{\prime \prime}(\zeta)}{2\left(f^{\prime}(\zeta)\right)^{2}}\right)^{2}}
\end{aligned}
$$

Notice that, due to the exponential decay of $w_{\text {ext }}(s), \Phi_{1}(f(\zeta)-f(\bar{\zeta}))$ decays exponentially as $(\bar{\zeta}-\zeta) \rightarrow \infty$, and $\left|\Phi_{1}(f(\zeta)-f(\bar{\zeta}))\right| \leq \frac{C}{f^{\prime}(\bar{\zeta})} \frac{1}{(\zeta-\bar{\zeta})^{3}}$ as $(\zeta-\bar{\zeta}) \rightarrow \infty$. Using the properties of $f(\zeta)$ in Appendix B it follows that:

$$
\int_{\zeta_{0}}^{\bar{\zeta}+\delta_{1}(\bar{\zeta})} \Phi_{1}(f(\zeta)-f(\bar{\zeta})) d \zeta \sim \frac{1}{f^{\prime}(\bar{\zeta})} \int_{-\infty}^{\infty} \Phi_{1}(s-f(\bar{\zeta})) d s \equiv \frac{a}{f^{\prime}(\bar{\zeta})}
$$

On the other hand, since $\beta(f(\zeta))$ decays like $\frac{1}{\left(f^{\prime}(\bar{\zeta})\right)^{1+\alpha}}$ with $0<\alpha<1$ we obtain that the term $I_{2}$ might be estimated in a similar manner. Similar estimates can be obtained for the derivatives of $I_{1}, I_{2}$ 
(cf. [17] ). Therefore, the equation (188) can be approximated as:

$$
\int_{\zeta_{0}}^{\bar{\zeta}+1} \frac{\sin ^{2}\left(\pi\left(\zeta-\bar{\zeta}-Z\left(\zeta, \bar{\zeta}, \zeta_{0}\right)\right)\right) \lambda(\zeta)}{\pi^{2}} d \zeta=\theta\left(\bar{\zeta}, \zeta_{0}\right)-\varepsilon(\bar{\zeta})
$$

where $|\varepsilon(\bar{\zeta})| \leq \frac{C}{f^{\prime}(\bar{\zeta})}$.

We have then reduced the study of the transition problem to the integral equation (192) Notice that (189) implies that the kernel $\frac{\sin ^{2}\left(\pi\left(\zeta-\bar{\zeta}-Z\left(\zeta, \bar{\zeta}, \zeta_{0}\right)\right)\right)}{\pi^{2}}$ becomes negligible as soon as $(W-1)$ becomes of order one. This suggests the following rough approximation for (192):

$$
\int_{\bar{\zeta}}^{\bar{\zeta}+1} \frac{\sin ^{2}\left(\pi\left(\zeta-\bar{\zeta}-Z\left(\zeta, \bar{\zeta}, \zeta_{0}\right)\right)\right) \lambda(\zeta)}{\pi^{2}} d \zeta=\theta\left(\bar{\zeta}, \zeta_{0}\right)-\varepsilon\left(\bar{\zeta}, \zeta_{0}\right)
$$

This equation is a nonhomogeneous version of (158). Notice that the right-hand side of (193) is bounded by $\frac{C}{\left(f^{\prime}(\bar{\zeta})\right)^{\alpha}}$, and similar estimates can be obtained for the derivatives (cf. [17]). Arguing then as in Subsection 4.4.2, and including in the error terms the contributions of $R$ in (180), we can transform (193) into

$$
\lambda(\bar{\zeta}+1)-\lambda(\bar{\zeta})=O\left(\frac{1}{\left(f^{\prime}(\bar{\zeta})\right)^{\alpha}}\right)
$$

as $\bar{\zeta} \rightarrow \infty$. This equation might be solved by iteration. Due to the fast growth of the function $f^{\prime}(\bar{\zeta})$ the resulting function $\lambda(\bar{\zeta})$ is globally bounded. More precisely, the function $\lambda(\bar{\zeta})$ approaches asymptotically to a periodic function with period one as $\bar{\zeta} \rightarrow \infty$. Due to (183), (184) it follows that $\tilde{\beta}(\tau)$ and $\bar{\beta}(\tau)$ differ in a very small function as $\tau \rightarrow \infty$.

The approximation of analysis of (192) by means of (193) must be made more carefully because the three differentiations of (193) require to differentiate (189) and this yields terms containing derivatives of $\lambda$ that must be examined in detail.

More precisely, differentiating (192) and using (189) we obtain, to the leading order:

$$
\frac{d\left(\theta\left(\bar{\zeta}, \zeta_{0}\right)-\varepsilon\left(\bar{\zeta}, \zeta_{0}\right)\right)}{d \bar{\zeta}}=\frac{9}{\left(f^{\prime}(\bar{\zeta}+1)\right)^{2}} \frac{\lambda(\bar{\zeta}+1)}{(\alpha-1)^{2}}+\int_{\zeta_{0}}^{\bar{\zeta}+1} \frac{d}{d \bar{\zeta}}\left(\frac{\sin ^{2}(\pi(\zeta-\bar{\zeta}-Z))}{\pi^{2}}\right) \lambda(\zeta) d \zeta
$$

Combining (189), (191) we derive the following approximation for $\frac{\sin ^{2}(\pi(\zeta-\bar{\zeta}-Z))}{\pi^{2}}$ in the region $\zeta \approx$ $\bar{\zeta}+1$ :

$$
\frac{\sin ^{2}(\pi(\zeta-\bar{\zeta}-Z))}{\pi^{2}} \sim \frac{9 \Omega\left(f(\zeta)-f(\zeta)-F_{\text {int }}(\alpha)\right)}{\left(f^{\prime}(\zeta)\right)^{2}}
$$

where:

$$
\Omega(s)=\frac{1}{\left(w_{\text {int }}(s)-1\right)^{2}}
$$

Therefore, we derive the following approximations, for the derivatives of $\frac{\sin ^{2}(\pi(\zeta-\bar{\zeta}-Z))}{\pi^{2}}$ in the region $\zeta \approx \bar{\zeta}+1:$

$$
\begin{aligned}
\frac{d}{d \bar{\zeta}}\left(\frac{\sin ^{2}(\pi(\zeta-\bar{\zeta}-Z))}{\pi^{2}}\right) & \sim-\frac{9 \Omega^{\prime}\left(f(\zeta)-f(\bar{\zeta}+1)-F_{\text {int }}(\alpha)\right)}{f^{\prime}(\zeta)} \\
\frac{d^{2}}{d \bar{\zeta}^{2}}\left(\frac{\sin ^{2}(\pi(\zeta-\bar{\zeta}-Z))}{\pi^{2}}\right) & \sim 9 \Omega^{\prime \prime}\left(f(\zeta)-f(\bar{\zeta}+1)-F_{\text {int }}(\alpha)\right) \\
\frac{d^{3}}{d \bar{\zeta}^{3}}\left(\frac{\sin ^{2}(\pi(\zeta-\bar{\zeta}-Z))}{\pi^{2}}\right) & \sim-9 f^{\prime}(\bar{\zeta}+1) \Omega^{\prime \prime}\left(f(\zeta)-f(\bar{\zeta}+1)-F_{\text {int }}(\alpha)\right)
\end{aligned}
$$


where we have used the properties of the function $f$ in Appendix B. Differentiating (195), using the previous approximations for the derivatives of $\frac{\sin ^{2}(\pi(\zeta-\bar{\zeta}-Z))}{\pi^{2}}$, and neglecting the smaller contributions multiplying the terms $\lambda(\bar{\zeta}+1)$ or its derivatives we arrive at:

$$
\begin{aligned}
& \frac{d^{2}\left(\theta\left(\bar{\zeta}, \zeta_{0}\right)-\varepsilon\left(\bar{\zeta}, \zeta_{0}\right)\right)}{d \bar{\zeta}^{2}} \\
& =\frac{9}{\left(f^{\prime}(\bar{\zeta}+1)\right)^{2}} \frac{\lambda^{\prime}(\bar{\zeta}+1)}{(\alpha-1)^{2}}-\frac{9 \Omega^{\prime}\left(-F_{\text {int }}(\alpha)\right)}{f^{\prime}(\bar{\zeta}+1)} \lambda(\bar{\zeta}+1)+ \\
& \int_{\zeta_{0}}^{\bar{\zeta}+1} \frac{d^{2}}{d \bar{\zeta}^{2}}\left(\frac{\sin ^{2}(\pi(\zeta-\bar{\zeta}-Z))}{\pi^{2}}\right) \lambda(\zeta) d \zeta \\
& \frac{d^{3}\left(\theta\left(\bar{\zeta}, \zeta_{0}\right)-\varepsilon\left(\bar{\zeta}, \zeta_{0}\right)\right)}{d \bar{\zeta}^{3}} \\
& =\frac{9}{\left(f^{\prime}(\bar{\zeta}+1)\right)^{2}} \frac{\lambda^{\prime \prime}(\bar{\zeta}+1)}{(\alpha-1)^{2}}-\frac{9 \Omega^{\prime}\left(-F_{\text {int }}(\alpha)\right)}{f^{\prime}(\bar{\zeta}+1)} \lambda^{\prime}(\bar{\zeta}+1)+9 \Omega^{\prime \prime}\left(-F_{\text {int }}(\alpha)\right) \lambda(\bar{\zeta}+1) \\
& +\int_{\zeta_{0}}^{\bar{\zeta}+1} \frac{d^{3}}{d \bar{\zeta}^{3}}\left(\frac{\sin ^{2}(\pi(\zeta-\bar{\zeta}-Z))}{\pi^{2}}\right) \lambda(\zeta) d \zeta
\end{aligned}
$$

We now derive approximations for the terms $\frac{d^{2}}{d \zeta^{2}}\left(\frac{\sin ^{2}(\pi(\zeta-\bar{\zeta}-Z))}{\pi^{2}}\right), \frac{d^{3}}{d \zeta^{3}}\left(\frac{\sin ^{2}(\pi(\zeta-\bar{\zeta}-Z))}{\pi^{2}}\right)$ in (196), (197). In order to compute an approximation for these terms in the region $\zeta \approx \bar{\zeta}$ we use (189), (190). Then:

$$
\frac{\sin ^{2}(\pi(\zeta-\bar{\zeta}-Z))}{\pi^{2}} \sim \frac{9 \Psi(f(\zeta)-f(\bar{\zeta}))}{\left(f^{\prime}(\zeta)\right)^{2}}
$$

and differentiating this formula we then arrive at:

$$
\begin{aligned}
\frac{d}{d \bar{\zeta}}\left(\frac{\sin ^{2}(\pi(\zeta-\bar{\zeta}-Z))}{\pi^{2}}\right) & \sim-\frac{9 \Psi^{\prime}(f(\zeta)-f(\bar{\zeta}))}{f^{\prime}(\zeta)} \\
\frac{d^{2}}{d \bar{\zeta}^{2}}\left(\frac{\sin ^{2}(\pi(\zeta-\bar{\zeta}-Z))}{\pi^{2}}\right) & \sim 9 \Psi^{\prime \prime}(f(\zeta)-f(\bar{\zeta})) \\
\frac{d^{3}}{d \bar{\zeta}^{3}}\left(\frac{\sin ^{2}(\pi(\zeta-\bar{\zeta}-Z))}{\pi^{2}}\right) & \sim-9 f^{\prime}(\zeta) \Psi^{\prime \prime}(f(\zeta)-f(\bar{\zeta}))
\end{aligned}
$$

On the other hand, in order to estimate the term $\frac{\sin ^{2}(\pi(\zeta-\bar{\zeta}-Z))}{\pi^{2}}$ in the region $\left[\bar{\zeta}+\delta_{1}(\bar{\zeta}), \bar{\zeta}+1-\delta_{2}(\bar{\zeta})\right]$ we will assume that the term $Z$ might be neglected and therefore

$$
\frac{\sin ^{2}(\pi(\zeta-\bar{\zeta}-Z))}{\pi^{2}} \sim \frac{\sin ^{2}(\pi(\zeta-\bar{\zeta}))}{\pi^{2}}
$$

This approximation is useful to simplify the computations. Its validity will be discussed below in 
detail. Using (198), (197) becomes:

$$
\begin{aligned}
& \frac{d^{3}\left(\theta\left(\bar{\zeta}, \zeta_{0}\right)-\varepsilon\left(\bar{\zeta}, \zeta_{0}\right)\right)}{d \bar{\zeta}^{3}} \\
& =\frac{9}{\left(f^{\prime}(\bar{\zeta}+1)\right)^{2}} \frac{\lambda^{\prime \prime}(\bar{\zeta}+1)}{(\alpha-1)^{2}}-\frac{9 \Omega^{\prime}\left(-F_{\text {int }}(\alpha)\right)}{f^{\prime}(\bar{\zeta}+1)} \lambda^{\prime}(\bar{\zeta}+1)+9 \Omega^{\prime \prime}\left(-F_{\text {int }}(\alpha)\right) \lambda(\bar{\zeta}+1) \\
& -9 \int_{\bar{\zeta}+1-\delta_{2}(\bar{\zeta})}^{\bar{\zeta}+1} f^{\prime}(\zeta) \Omega^{\prime \prime \prime}\left(f(\zeta)-f(\bar{\zeta}+1)-F_{\text {int }}(\alpha)\right) \lambda(\zeta) d \zeta \\
& -9 \int_{\zeta_{0}}^{\bar{\zeta}+\delta_{1}(\bar{\zeta})} f^{\prime}(\zeta) \Psi^{\prime \prime \prime}(f(\zeta)-f(\bar{\zeta})) \lambda(\zeta) d \zeta- \\
& -4 \pi^{2} \int_{\bar{\zeta}}^{\bar{\zeta}+1} \sin (2 \pi(\zeta-\bar{\zeta})) \lambda(\zeta) d \zeta
\end{aligned}
$$

In order to estimate the term $\int_{\bar{\zeta}}^{\bar{\zeta}+1} \sin (2 \pi(\zeta-\bar{\zeta})) \lambda(\zeta) d \zeta$ in (199) we use similar approximations in (195). Plugging the resulting formula into (199) to obtain:

$$
\begin{aligned}
& =\frac{1}{\left(f^{\prime}(\bar{\zeta}+1)\right)^{2}} \frac{\lambda^{\prime \prime}(\bar{\zeta}+1)}{(\alpha-1)^{2}}-\frac{\Omega^{\prime}\left(-F_{\text {int }}(\alpha)\right)}{f^{\prime}(\bar{\zeta}+1)} \lambda^{\prime}(\bar{\zeta}+1)+\Omega^{\prime \prime}\left(-F_{\text {int }}(\alpha)\right) \lambda(\bar{\zeta}+1) \\
& -\int_{\zeta_{0}}^{\bar{\zeta}+1} f^{\prime}(\zeta) \Omega^{\prime \prime \prime}\left(f(\zeta)-f(\bar{\zeta}+1)-F_{\text {int }}(\alpha)\right) \lambda(\zeta) d \zeta \\
& -\int_{\zeta_{0}}^{\bar{\zeta}+\delta_{1}(\bar{\zeta})} f^{\prime}(\zeta) \Psi^{\prime \prime \prime}(f(\zeta)-f(\bar{\zeta})) \lambda(\zeta) d \zeta= \\
& =O\left(\frac{1}{(f(\bar{\zeta}))^{\alpha}}\right)
\end{aligned}
$$

as $\bar{\zeta} \rightarrow \infty$. We have used the fast decay of the term $\Omega^{\prime \prime \prime}(s)$, in order to replace the lower extreme of integration in the first integral by $\zeta_{0}$.

The integrodifferential equation (200) is the basic equation that describes the evolution of the function $\lambda(\zeta)$, or equivalently $\bar{\beta}(\tau)$. This equation has two different time scales. Indeed, since $\frac{d}{d \tau}=\frac{1}{f^{\prime}(\zeta)} \frac{d}{d \zeta}$, it follows that the natural time scale for the terms containing the derivatives is $\delta \zeta \approx \frac{1}{f(\bar{\zeta}+1)}$, or equivalently $\delta \tau \approx 1$. On the other hand, the integral terms might be approximated, for functions $\lambda$ that are approximately constant in the time scale $\tau$ as Dirac masses in $\zeta=\bar{\zeta}, \zeta=\bar{\zeta}+1$ respectively. In particular these terms alone would tranform (200) in a delay equation of the form (194). In the original time variable $\tau$ this delay equation would relate the values of $\lambda$ at times $\tau$ and $S(\tau)$. In particular, under the assumption (153), $S(\tau)$ varies in a very slow, "adiabatic" manner in the time scale $\tau$. It is shown in Appendix $\mathrm{C}$ that, if the second integral term is frozen, the remaining terms in (200) yield stabilization to steady states in times $\delta \zeta \approx \frac{1}{f(\bar{\zeta}+1)}$, or equivalently in times $\delta \tau \approx 1$. This implies that the function $\lambda$ becomes approximately constant in time intervals or size $\delta \tau \approx 1$. Therefore, the integral terms might be approximated as Dirac masses and (200) becomes (194). The amount of mass concentrated in the Dirac masses can be computed using:

$$
\Omega^{\prime \prime}\left(-F_{\mathrm{int}}(\alpha)\right)-9 \int_{-\infty}^{0} \Omega^{\prime \prime \prime}\left(s-F_{\mathrm{int}}(\alpha)\right) d s=\int_{-\infty}^{\infty} \Psi^{\prime \prime \prime}(s) d s=\frac{2}{9}
$$

Let us discuss now the validity of the assumption (198). Notice that due to (180)-(182) the order of magnitude of $Z$ is the same as the one of $\lambda$ in the region where $\zeta-\bar{\zeta}$ is of order one. Nevertheless, due to the arbitrariness of $f(\zeta)$ would be possible to choose $\lambda$ small for large $\zeta$ and (194) would imply that 
$\lambda$ is small for large $\zeta$. In any case, since the order of magnitude of $\lambda$ is one, the argument above is not completely rigorous. However, the argument could be made keeping the term $Z$ in (198) in the derivation of (200). In particular this would introduce several additional terms containing $Z$ and its derivatives similar to the ones in Subsection 4.4.2. Detailed, although more technical computations, following this approach can be found in [17]. Nevertheless, the final result is again (200). Therefore the analysis above can be kept without major changes.

It is interesting to discuss the meaning of the integrodifferential equation (200) containing two different time scales. The time scale $(\bar{\tau}-\tau)$ of order in which stabilization takes place is associated to the effect of the characteristics that are placed in the region $W \in[0,1)$. These characteristics yield stabilization of the dynamics of the LSW model to the simplified dynamics (194). This simplified equation indicates that in some sense, the solutions of the transition problem (174)-(176) are able to "see" the values of the function $\bar{\beta}(\bar{\tau})$ at the times $\tau=S(\bar{\tau})$, that is the time when the characteristic that is at $W=\alpha$ for $\tau=\bar{\tau}$ arrived to that critical line. Actually, the analysis above and in particular equation (194) indicates that these are the only times when the values of $\beta$ are relevant as $\tau \rightarrow \infty$ for solutions behaving in a self-similar manner.

\subsection{Linearizing the LSW model near self-similar solutions}

In this Section we will use ideas analogous to those in previous subsection to linearize near self-similar solutions the whole LSW model and not just the transition problem (174)-(176).

We notice that the volume conservation condition (12) condition equivales to

$$
\int_{0}^{\infty} G(W, \tau) d W=e^{\tau} \int_{0}^{\infty} G_{0}(W) d W
$$

On the other hand, using (114), (176) we obtain

$$
\int_{0}^{1} G_{0}\left(\bar{W}_{0}\left(\tau+F_{\text {int }}(W)-F_{\text {int }}(\alpha), \alpha\right)\right) d W=e^{\tau} \int_{0}^{\infty} G_{0}(W) d W
$$

Combining (201), (202) we obtain:

$$
\int_{0}^{1}\left[G_{0}\left(W_{0}(\tau, W)\right)-G_{0}\left(\bar{W}_{0}\left(\tau+F_{\text {int }}(W)-F_{\text {int }}(\alpha), \alpha\right)\right)\right] d W=-\int_{1}^{\infty} G_{0}\left(W_{0}(\tau, W)\right) d W
$$

or, in an equivalent manner:

$$
\int_{0}^{1} e^{-F_{\text {int }}(W)}\left[\frac{G_{0}\left(W_{0}(\tau, W)\right)}{G_{0}\left(\bar{W}_{0}\left(\tau+F_{\text {int }}(W)-F_{\text {int }}(\alpha), \alpha\right)\right)}-1\right] d W=-\varepsilon(\tau)
$$

where

$$
\varepsilon(\tau) \equiv e^{\tau} \int_{1}^{\infty} \frac{G_{0}\left(W_{0}(\tau, W)\right)}{\int_{0}^{\infty} G_{0}(W) d W} d W
$$

We will assume in the remaining of this Subsection, that the initial data $G_{0}$ satisfies the assumption (150), (151). Under this assumption, equation (203) becomes, to the leading order:

$$
\begin{aligned}
& \int_{0}^{1} e^{-F_{\text {int }}(W)} . \\
& {\left[\exp \left(-\frac{W_{0}(\tau, W)-\bar{W}_{0}\left(\tau+F_{\text {int }}(W)-F_{\text {int }}(\alpha), \alpha\right)}{\lambda\left(w_{\text {ext }}^{-1}\left(\bar{W}_{0}\left(\tau+F_{\text {int }}(W)-F_{\text {int }}(\alpha), \alpha\right)\right)\right) \bar{W}_{0}\left(\tau+F_{\text {int }}(W)-F_{\text {int }}(\alpha), \alpha\right)}\right)-1\right] d W} \\
& =-\varepsilon(\tau)
\end{aligned}
$$


where we are using (116), (117) and where, from now on, we will include in $\varepsilon(\tau)$ additional corrective terms that converge to zero as $\tau \rightarrow \infty$. Notice that in the derivation of (204) we assume that the term inside the exponential is bounded. Moreover, we will assume also that $\frac{W_{0}(\tau, W)}{W_{0}\left(\tau+F_{\text {int }}(W)-F_{\text {int }}(\alpha), \alpha\right)} \rightarrow 1$. These assumptions will be justified "a posteriori" in a self-consistent manner, checking that the equations derived with these assumptions imply them. Linearizing then the exponential term we arrive at:

$$
\int_{0}^{1} \frac{e^{-F_{\text {int }}(W)}}{\lambda\left(w_{\text {ext }}^{-1}\left(\bar{W}_{0}\left(\tau+F_{\text {int }}(W)-F_{\text {int }}(\alpha), \alpha\right)\right)\right)}\left(\frac{W_{0}(\tau, W)}{\bar{W}_{0}\left(\tau+F_{\text {int }}(W)-F_{\text {int }}(\alpha), \alpha\right)}-1\right) d W=\varepsilon(\tau)
$$

and, neglecting quadratic terms on $\left(W_{0}(\tau, W)-\bar{W}_{0}\left(\tau+F_{\text {int }}(W)-F_{\text {int }}(\alpha), \alpha\right)\right)$.

$$
\begin{aligned}
& \int_{0}^{1} \frac{e^{-F_{\text {int }}(W)}}{\lambda\left(w_{\text {ext }}^{-1}\left(\bar{W}_{0}\left(\tau+F_{\text {int }}(W)-F_{\text {int }}(\alpha), \alpha\right)\right)\right)}\left(\frac{W_{0}(\tau, W)}{\bar{W}_{0}(\tau, W)}-1\right) d W \\
& \sim \int_{0}^{1} \frac{e^{-F_{\text {int }}(W)}}{\lambda\left(w_{\text {ext }}^{-1}\left(\bar{W}_{0}\left(\tau+F_{\text {int }}(W)\right)-F_{\text {int }}(\alpha), \alpha\right)\right)}\left(\frac{\bar{W}_{0}(\tau, W)}{\bar{W}_{0}\left(\tau+F_{\text {int }}(W)-F_{\text {int }}(\alpha), \alpha\right)}-1\right) d W \\
& =\varepsilon(\tau)
\end{aligned}
$$

In order to estimate the second term on the left-hand side of (206) we define a new variable $\eta$ such that:

$$
\bar{W}_{0}(\bar{\tau}, W)=\bar{W}_{0}\left(\bar{\tau}+F_{\text {int }}(W)-F_{\text {int }}(\alpha), \alpha+\eta\right)
$$

Notice that, since to the leading order:

$$
\tau+F_{\text {int }}\left(W\left(\tau, \bar{W}_{0}(\bar{\tau}, \bar{W})\right)\right)=\bar{\tau}+F_{\text {int }}(\bar{W})
$$

it follows that $\eta$ is small. Moreover, since the correction to this approximation is due to the term $\bar{\beta}$, it follows that:

$$
|\eta| \leq C|\bar{\beta}(\bar{\tau})|
$$

We can now estimate a rough estimate of $\frac{\bar{W}_{0}(\tau, W)-\bar{W}_{0}\left(\tau+F_{\text {int }}(W)-F_{\text {int }}(\alpha), \alpha\right)}{\bar{W}_{0}\left(\tau+F_{\text {int }}(W)-F_{\text {int }}(\alpha), \alpha\right)}$ using the fact that to the leading order $\log \left(\bar{W}_{0}(\tau, W)\right) \sim S(\tau)$, (cf. (131)). Then:

$$
\begin{aligned}
& \frac{\bar{W}_{0}(\tau, W)-\bar{W}_{0}\left(\tau+F_{\text {int }}(W)-F_{\text {int }}(\alpha), \alpha\right)}{\bar{W}_{0}\left(\tau+F_{\text {int }}(W)-F_{\text {int }}(\alpha), \alpha\right)} \\
& =\frac{\bar{W}_{0}\left(\bar{\tau}+F_{\text {int }}(W)-F_{\text {int }}(\alpha), \alpha+\eta\right)-\bar{W}_{0}\left(\tau+F_{\text {int }}(W)-F_{\text {int }}(\alpha), \alpha\right)}{\bar{W}_{0}\left(\tau+F_{\text {int }}(W)-F_{\text {int }}(\alpha), \alpha\right)} \\
& \approx \frac{\frac{\partial}{\partial W}\left(\bar{W}_{0}\left(\tau+F_{\text {int }}(W)-F_{\text {int }}(\alpha), \alpha\right)\right)}{\bar{W}_{0}\left(\tau+F_{\text {int }}(W)-F_{\text {int }}(\alpha), \alpha\right)} \eta \sim S^{\prime}(\tau) \eta=O\left(\frac{|\bar{\beta}(\tau)|}{\tau}\right)
\end{aligned}
$$

and:

$$
\begin{aligned}
& \tilde{\varepsilon}(\tau) \\
& \equiv\left|\int_{0}^{1} \frac{e^{-F_{\text {int }}(W)}}{\lambda\left(w_{\text {ext }}^{-1}\left(\bar{W}_{0}\left(\tau+F_{\text {int }}(W)\right)-F_{\text {int }}(\alpha), \alpha\right)\right)}\left(\frac{\bar{W}_{0}(\tau, W)}{\bar{W}_{0}\left(\tau+F_{\text {int }}(W)-F_{\text {int }}(\alpha), \alpha\right)}-1\right) d W\right| \\
& \leq \frac{C|\bar{\beta}(\tau)|}{\tau \lambda\left(w_{\text {ext }}^{-1}\left(\bar{W}_{0}(\tau, \alpha)\right)\right)}
\end{aligned}
$$

Finally, we estimate the first term in (206). To this end we use again the change of variables (177)(179). Let us denote from now on as $\bar{\lambda}(\zeta), \bar{Z}\left(\zeta, \bar{\zeta}, \zeta_{0}\right)$ the approximated solution constructed in Subsection 4.4.3. Let us write $U \equiv Z-\bar{Z}$. Linearizing in (180)-(182) it follows that:

$$
\begin{aligned}
U_{\zeta} & =a(\zeta) U+\frac{\sin ^{2}(\pi(\zeta-\bar{\zeta}-\bar{Z}))}{\pi^{2}} \sigma(\zeta) \\
U(\bar{\zeta}+1) & =0
\end{aligned}
$$


where

$$
\left.a(\zeta) \equiv \frac{\partial}{\partial Z}\left(\frac{\sin ^{2}(\pi(\zeta-\bar{\zeta}-\bar{Z}))}{\pi^{2}}\right)\right|_{Z=\bar{Z}} \bar{\lambda}(\zeta)+\left.\frac{\partial}{\partial Z}\left(\frac{\sin ^{2}(\pi(\zeta-\bar{\zeta}-\bar{Z})) R(Z, \zeta, \bar{\zeta})}{\pi^{2}}\right)\right|_{Z=\bar{Z}}
$$

Our goal is to estimate $U\left(\zeta_{0}\right)$, that is given by:

$$
U\left(\zeta_{0}\right)=-\int_{\zeta_{0}}^{\bar{\zeta}+1} e^{\int_{\eta}^{\bar{\zeta}+1} a(\xi) d \xi} \frac{\sin ^{2}(\pi(\zeta-\bar{\zeta}-\bar{Z}))}{\pi^{2}} \sigma(\zeta) d \zeta
$$

It turns out that the integral term in (207) is small, because the term containing $R(Z, \zeta, \bar{\zeta})$ might be estimated as in Subsection 4.4.3, and the term containing $\bar{\lambda}(\zeta)$ can be estimated using the fact that $\bar{\lambda}$ is small. Therefore, to the leading order:

$$
U\left(\zeta_{0}\right)=-\int_{\zeta_{0}}^{\bar{\zeta}+1} \frac{\sin ^{2}(\pi(\zeta-\bar{\zeta}-\bar{Z}))}{\pi^{2}} \sigma(\zeta) d \zeta
$$

We now recall that our goal is to estimate the term in (206). To this end we use the change of variables (178), (179) that yields:

$$
\begin{aligned}
& W_{0}(\tau, W)=1+\frac{3 \pi \cot (\pi(\zeta-\bar{\zeta}-\bar{Z}-U))}{f^{\prime}(\zeta)}+\frac{3 f^{\prime \prime}(\zeta)}{2\left(f^{\prime}(\zeta)\right)^{2}} \text { at } \zeta=\zeta_{0} \\
& \bar{W}_{0}(\tau, W)=1+\frac{3 \pi \cot (\pi(\zeta-\bar{\zeta}-\bar{Z}))}{f^{\prime}(\zeta)}+\frac{3 f^{\prime \prime}(\zeta)}{2\left(f^{\prime}(\zeta)\right)^{2}} \text { at } \zeta=\zeta_{0}
\end{aligned}
$$

In order to compute the difference $\left(W_{0}(\tau, W)-\bar{W}_{0}(\tau, W)\right)$ we use the trigonometric formula $\cot (A-B)-$ $\cot (A)=\frac{1+\cot ^{2}(A)}{\cot (B)-\cot (A)}$. Then, after some computations we obtain:

$$
\begin{aligned}
& \int_{0}^{1} \frac{e^{-F_{\text {int }}(W)}}{\lambda\left(w_{\text {ext }}^{-1}\left(\bar{W}_{0}\left(\tau+F_{\mathrm{int}}(W)-F_{\text {int }}(\alpha), \alpha\right)\right)\right)}\left(\frac{W_{0}(\tau, W)}{\bar{W}_{0}(\tau, W)}-1\right) d W \\
& =\int_{0}^{1} \frac{e^{-F_{\text {int }}(W)}}{\lambda\left(w_{\text {ext }}^{-1}\left(\bar{W}_{0}\left(\tau+F_{\text {int }}(W)-F_{\text {int }}(\alpha), \alpha\right)\right)\right)} \frac{\cot \left(\pi\left(\zeta_{0}-\bar{\zeta}-\bar{Z}\right)\right)}{\cot (\pi(\zeta-\bar{\zeta}-\bar{Z}))-\cot (\pi U)} d W \\
& =\varepsilon(\tau)-\tilde{\varepsilon}(\tau)
\end{aligned}
$$

Notice that $\pi\left(\zeta_{0}-\bar{\zeta}-\bar{Z}\right)<<1$. On the other hand we will assume, something to be checked "a posteriori" that $|U|<<\left(\zeta_{0}-\bar{\zeta}-\bar{Z}\right)$. Then $\cot (\pi U)>>\cot (\pi(\zeta-\bar{\zeta}-\bar{Z}))$, whence, to the leading order

$$
\int_{0}^{1} \frac{e^{-F_{\text {int }}(W)}}{\lambda\left(w_{\text {ext }}^{-1}\left(\bar{W}_{0}\left(\tau+F_{\text {int }}(W)-F_{\text {int }}(\alpha), \alpha\right)\right)\right)} \frac{\cot \left(\pi\left(\zeta_{0}-\bar{\zeta}-\bar{Z}\right)\right)}{\cot (\pi U)} d W=-\varepsilon(\tau)+\tilde{\varepsilon}(\tau)
$$

Using the formula $\cot (x) \sim \frac{1}{x}$ as $x \rightarrow 0$, as well as the approximation $\frac{\cot \left(\pi\left(\zeta_{0}-\bar{\zeta}-\bar{Z}\left(\zeta_{0}, \bar{\zeta}, W\right)\right)\right)}{\lambda\left(w_{\text {ext }}^{-1}\left(\bar{W}_{0}\left(\tau+F_{\text {int }}(W)-F_{\text {int }}(\alpha), \alpha\right)\right)\right)} \sim$ $\frac{\cot \left(\pi\left(\zeta_{0}-\bar{\zeta}-\bar{Z}\left(\zeta_{0} \bar{\zeta}, \alpha\right)\right)\right)}{\lambda\left(w_{\text {ext }}^{-1}\left(\bar{W}_{0}(\tau, \alpha)\right)\right)}$ it follows that:

$$
\begin{aligned}
& \int_{0}^{1}\left[e^{-F_{\mathrm{int}}(W)} U\left(\zeta_{0}, \bar{\zeta}, W\right)\right] d W \\
& =\frac{[-\varepsilon(\tau)+\tilde{\varepsilon}(\tau)] \lambda\left(w_{\mathrm{ext}}^{-1}\left(\bar{W}_{0}(\tau, \alpha)\right)\right)}{\pi \cot \left(\pi\left(\zeta_{0}-\bar{\zeta}-\bar{Z}\left(\zeta_{0}, \bar{\zeta}, \alpha\right)\right)\right)} \equiv-v(\tau)
\end{aligned}
$$


where $U$ is given by $(208)$ and where, from now on, $v(\tau)$ is a generic function satisfying $v(\tau)=O\left(\frac{1}{(f(\zeta))^{\beta}}\right)$ as $\zeta \rightarrow \infty$, for some $\beta>0$. Then:

$$
\int_{\zeta_{0}}^{\bar{\zeta}+1}\left[\int_{0}^{1} e^{-F_{\mathrm{int}}(W)} \frac{\sin ^{2}\left(\pi\left(\zeta-\bar{\zeta}-\bar{Z}\left(\zeta_{0}, \bar{\zeta}, W\right)\right)\right)}{\pi^{2}} d W\right] \sigma(\zeta) d \zeta=v(\tau)
$$

This equation can be studied basically in the same manner as (192) with the only difference that there is an integration on the variable $W$, instead of just a particular choice of $\alpha$.

Equation (209) can be analyzed in a manner analogous to (192). Arguing as in the derivation of (200) we obtain:

$$
\begin{aligned}
& \frac{d^{3}(v)}{d \bar{\zeta}^{3}}+4 \pi^{2} \frac{d}{d \bar{\zeta}}(v) \\
& =\frac{9 \lambda^{\prime \prime}(\bar{\zeta}+1)}{\left(f^{\prime}(\bar{\zeta}+1)\right)^{2}} \int_{0}^{1} \frac{e^{-F_{\mathrm{int}}(W)} d W}{(W-1)^{2}}-\frac{9 \lambda^{\prime}(\bar{\zeta}+1)}{f^{\prime}(\bar{\zeta}+1)} \int_{0}^{1} e^{-F_{\mathrm{int}}(W)} \Omega^{\prime}\left(-F_{\mathrm{int}}(W)\right) d W \\
& +9 \lambda(\bar{\zeta}+1) \int_{0}^{1} e^{-F_{\mathrm{int}}(W)} \Omega^{\prime \prime}\left(-F_{\mathrm{int}}(W)\right) d W \\
& -9 \int_{\zeta_{0}}^{f^{\prime}(\zeta)}\left[\int_{0}^{1} e^{-F_{\mathrm{int}}(W)} \Omega^{\prime \prime \prime}\left(f(\zeta)-f(\bar{\zeta}+1)-F_{\mathrm{int}}(W)\right) d W\right] \lambda(\zeta) d \zeta \\
& -9 \int_{\zeta_{0}}^{\bar{\zeta}+\delta_{1}(\bar{\zeta})} f^{\prime}(\zeta) \Psi^{\prime \prime \prime}\left(f(\zeta)-f\left(f^{-1}\left(f(\bar{\zeta}+1)+F_{\mathrm{int}}(W)-F_{\mathrm{int}}(\alpha)\right)-1\right)\right) \lambda(\zeta) d \zeta
\end{aligned}
$$

Equation (210) might be analysed exactly as (200) In Subsection 4.4.3. As in that case this equation has two different time scales. The shorter time scale is of order $\frac{1}{f^{\prime}(\bar{\zeta}+1)}$ and is the scale associated to the terms containing derivatives on $\lambda$ as well as the integral terms. The resulting operators yield stable behaviour as proved in Appendix C. Then, in the longer time scale where $\bar{\zeta}$ varies quantities of order one we can approximate (210) as in Subsection 4.4.3 to obtain:

$$
\lambda(\bar{\zeta}+1)-\lambda(\bar{\zeta})=\frac{1}{2}\left[\frac{d^{3}(v)}{d \bar{\zeta}^{3}}+4 \pi^{2} \frac{d}{d \bar{\zeta}}(v)\right]
$$

and the desired stability of the self-similar behaviour follows.

\section{CONCLUDING REMARKS}

In this paper, a formalism that allows to approximate asymptotically the noncompactly supported solutions of the LSW that approach asymptotically to self-similar solutions has been developed. An analogous, although fully rigorous analysis was made in [13], [14] for compactly supported initial data. As in the case of compactly supported initial data we have obtained that the resulting LSW dynamics might be approximated by means of integro-differential equations. There are, however, several crucial differences between both cases. In the compactly supported case, at a given time, the only particles that are relevant in the description of the long time asymptotics of the solutions are those who are vanishing in a time of order one. On the contrary, in the noncompactly supported case, there are two groups of particles playing a relevant role in the description of the dynamics of the LSW model near self-similar solutions. A first group is the set of particles whose remaining life-time is of order one, exactly as in the compactly supported case. On the other hand, the subset of the family of particles with a long life-time expectancy, whose radii is close to the so-called critical radius plays also a relevant role in the dynamics of the LSW 
system for noncompactly supported data. This fact does not have a natural correspondence in the compactly supported case. From the mathematical point of view, the main consequence is that, for compactly supported solutions, the LSW dynamics might be approximated by means of a set of integral equations of convolution type with fast decaying kernels. In the noncompactly supported case the final equations describing the evolution of the solutions are integro-differential equations having two time scales that can be studied using multiple scale methods.

The analysis in this paper shows that there exists a large class of noncompactly supported initial data yielding self similar behaviours for long times. However, it has been also shown that not all the initial data yield such a self-similar behaviour.

Acknowledgements: I thank B. Niethammer for several illuminating discussions during the realization of this work. This work is partially supported by DGES Project MTM2004-05634.

\section{References}

[1] J. Carr and O. Penrose. Asymptotic behaviour in a simplified Lifshitz-Slyozov equation. Physica D, 124:166-176, (1998).

[2] J. Carrillo and T. Goudon. A numerical study on large-time asymptotics of the Lifshitz-Slyozov system. J. Sc. Comp., 20 1:69-113, (2004).

[3] A. Garroni and B. Niethammer. Correctors and error-estimates in the homogenization of a MullinsSekerka problem. Annales de l'IHP: Analyse Nonlineare, 194, 371-393, (2002).

[4] E. Hille, Ordinary differential equations in the Complex Domain, Dover Publications Inc. 1997.

[5] I. M. Lifshitz and V. V. Slyozov, The kinetics of precipitation from supersaturated solid solutions. J. Phys. Chem. Solids, 19: 35-50, (1961).

[6] B. Giron, B. Meerson and P. V. Sasorov. Weak selection and stability of localized distributions in Ostwald ripening. Phys. Rev. E, 58:4213-6, (1998).

[7] B. Niethammer. Derivation of the LSW theory for Ostwald ripening by homogenization methods. Arch. Rat. Mech. Anal. 147(2), 119-178, (1999).

[8] B. Niethammer and F. Otto. Ostwald ripening: The screening length revisited. Calc. Var. 13(1), 33-68, (2001).

[9] B. Niethammer and R. L. Pego. Non-self-similar behaviour in the LSW theory of Ostwald ripening. J. Stat. Phys., 95, 5/6:867-902, (1999).

[10] B. Niethammer and R. L. Pego. On the initial value problem in the Lifshitz-Slyozov-Wagner theory of Ostwald ripening. SIAM J. Math. Anal., 31,3:457-485, (2000).

[11] B. Niethammer and R. L. Pego. Well-posedness for measure transport in a family of nonlocal domain coarsening models. Indiana Univ. Math. J., 54, 2:499-530, (2005).

[12] B. Niethammer and J. J. L. Velázquez. Screening in interacting particle systems. Arch. Rat. Mech. Anal. To appear

[13] B. Niethammer and J. J. L. Velázquez. Global stability and bounds for coarsening rates within the LSW mean-field theory for domain coarsening. Preprint.

[14] B. Niethammer and J. J. L. Velázquez. On the long time behaviour of the LSW model. Preprint.

[15] J. J. L. Velázquez, The Becker-Döring equations and the Lifshitz-Slyozov theory of coarsening. J. Stat. Phys. 92, 1/2, 195-236, (1998). 
[16] J. J. L. Velázquez, On the effect of stochastic fluctuations in the dynamics of the LSW theory of coarsening. J. Stat. Phys. 99, 1/2: 57-113, (2000).

[17] J. J. L. Velázquez, On the dynamics of the characteristic curves for the LSW model. Preprint.

[18] C. Wagner. Theorie der Alterung von Niederschlägen durch Umlösen. Z. Elektrochemie, 65:581-594, (1961).

[19] E. Zeidler. Nonlinear Functional Analysis and Its Applications. New York, Springer-Verlag, 1989.

\section{APPENDIX A: A LOCAL EXISTENCE THEOREM FOR THE TRANSITION PROBLEM}

In this Appendix we prove that the transition problem (111)-(115) is locally solvable in time.

Theorem 2 Let us define $W\left(s ; W_{0}\right)$ as the solution of the problem:

$$
\begin{aligned}
W_{s} & =-2+3 W^{1 / 3}-W+3 \beta(s) W^{1 / 3} \\
W\left(0 ; W_{0}\right) & =W_{0}
\end{aligned}
$$

Then, for a given function $f(\cdot) \in C^{1}[0, \delta]$ for some $\delta>0$ and satisfying $f(0)=\alpha, \alpha \in(0,1)$ there exists an unique continuous function $\beta(\cdot)$ defined in $s \in[0, T]$, for some $T>0$ such that:

$$
W(\tau ; f(\tau))=\alpha
$$

for $\tau \in[0, T]$. Moreover, suppose that for some $\bar{W}_{0}>0$ and $\beta(s)$ defined in $s \in[0, \bar{\tau}]$ the function $W\left(s ; \bar{W}_{0}\right)$ is defined and it remains positive in the interval $s \in[0, \bar{\tau}]$. Then, for any function $f(\cdot) \in$ $C^{1}[\bar{\tau}, \bar{\tau}+\delta]$ for some $\delta>0$, there exists $\beta(\cdot)$ defined in $s \in[\bar{\tau}, \bar{\tau}+T]$ such that (214) holds for $\tau \in$ $[\bar{\tau}, \bar{\tau}+T]$.

Proof. It reduces to a fixed point argument. Integrating both sides of (212) with $W_{0}=f(\tau)$ we obtain:

$$
W(\tau ; f(\tau))-f(\tau)=\int_{0}^{\tau}\left[-2+3(W(s ; f(\tau)))^{1 / 3}-W(s ; f(\tau))+3 \beta(s)(W(s ; f(\tau)))^{1 / 3}\right] d s
$$

Using (214) this equation becomes:

$$
\alpha-f(\tau)=\int_{0}^{\tau}\left[-2+3(W(s ; f(\tau)))^{1 / 3}-W(s ; f(\tau))+3 \beta(s)(W(s ; f(\tau)))^{1 / 3}\right] d s
$$

Differentiating (215) and using again (214) we obtain:

$$
\begin{aligned}
-f^{\prime}(\tau) & =\left[-(2+\alpha)+3(\alpha)^{1 / 3}\right]+3(\alpha)^{1 / 3} \beta(\tau)+ \\
& +f^{\prime}(\tau) \int_{0}^{\tau}\left[(W(s ; f(\tau)))^{-2 / 3}-1+\beta(s)(W(s ; f(\tau)))^{-2 / 3}\right] \frac{\partial W(s ; f(\tau))}{\partial W_{0}} d s
\end{aligned}
$$

Differentiating (212), (213) with respect to $W_{0}$ and integrating the resulting differential equation we obtain:

$$
\frac{\partial W(s ; f(\tau))}{\partial W_{0}}=\exp \left(\int_{0}^{s}\left[(W(\xi ; f(\tau)))^{-2 / 3}-1+\beta(\xi)(W(\xi ; f(\tau)))^{-2 / 3}\right] d \xi\right)
$$


and plugging this formula into (216) we obtain the equation:

$$
\begin{aligned}
& 3 \alpha^{1 / 3} \beta(\tau)+ \\
& f^{\prime}(\tau) \int_{0}^{\tau}\left[(W(s ; f(\tau)))^{-2 / 3}-1+\beta(s)(W(s ; f(\tau)))^{-2 / 3}\right] . \\
& \cdot e^{\int_{0}^{s}\left[(W(\xi ; f(\tau)))^{-2 / 3}-1+\beta(\xi)(W(\xi ; f(\tau)))^{-2 / 3}\right] d \xi} d s \\
& =-f^{\prime}(\tau)+\left[(2+\alpha)-3(\alpha)^{1 / 3}\right]
\end{aligned}
$$

where:

$$
W(s ; f(\tau))=f(\tau)+\int_{0}^{\tau}\left[-2+3(W(s ; f(\tau)))^{1 / 3}-W(s ; f(\tau))+3 \beta(s)(W(s ; f(\tau)))^{1 / 3}\right] d s
$$

The equation (217), (218) can be solved using a contractive fixed point argument on the space $\beta(\cdot) \in$ $C[0, T]$ with the uniform norm.

\section{APPENDIX B: SOME PROPERTIES OF THE FUNCTION $f(\zeta)$.}

In this Appendix we collect several properties of the function $f(\zeta)$ that have been used repeatedly in Section 4 . The function $f(\zeta)$ is defined by means of (134), (135) as well as the compatibility conditions (137)-(140). Although the properties described in this Appendix could be generalized to more general functions $S$, we will assume by definiteness that (153) holds, as well as similar asymptotic formulae for the derivatives of $S$. Under these assumptions the function $f$ has the following properties

$$
f(\zeta)>>\exp (\exp (\exp (\ldots \exp (\zeta)))) \quad \text { as } \zeta \rightarrow \infty
$$

for any finite number of iterated exponentials.

$$
f^{(k-1)}(\zeta)<<f^{(k)}(\zeta)<<(f(\zeta))^{1+\varepsilon} \quad \text { as } \zeta \rightarrow \infty
$$

for any $\epsilon>0$, and any $k=1,2,3$.

$$
f(\zeta+\delta)>>f(\zeta) \text { as } \zeta \rightarrow \infty
$$

for any $\delta>0$.

$$
f\left(\zeta+\frac{C}{f(\zeta)}\right) \sim f(\zeta) \quad \text { as } \zeta \rightarrow \infty
$$

for any $C>0$.

The property (219) just follows iterating (134) more than $k$ times. Property (221) can be proved in a similar manner. Indeed, iterating (134) by means of an exponential function it follows that,since $f(\zeta) \rightarrow \infty$, that $f(\zeta+\delta)-f(\zeta) \rightarrow \infty$, Then

$$
\begin{aligned}
f(\zeta+\delta) & =S^{-1}(f(\zeta+\delta-1))=S^{-1}([f(\zeta+\delta-1)-f(\zeta-1)]+f(\zeta-1)) \\
& >>S^{-1}(f(\zeta-1))=f(\zeta)
\end{aligned}
$$

as $\zeta \rightarrow \infty$.

Property (220) follows differentiating (134) that yields

$$
f^{\prime}(\zeta)=S^{\prime}(f(\zeta+1)) f^{\prime}(\zeta+1) \sim \frac{a}{f(\zeta+1)} f^{\prime}(\zeta+1)
$$


Iterating (223) to estimate $f^{\prime}(\zeta+1)$ it follows from $(219)$ that $f^{\prime}(\zeta) \rightarrow \infty$. Combining this with (223) we obtain the first inequality in (220) with $k=1$. On the other hand, combining (134) and (223) we obtain

$$
\frac{f^{\prime}(\zeta+1)}{f(\zeta+1)}=\left[\frac{1}{S^{\prime}(f(\zeta+1))} \frac{f(\zeta)}{S^{-1}(f(\zeta))}\right] \frac{f^{\prime}(\zeta)}{f(\zeta)}
$$

The term between brackets is bounded by $C f(\zeta)$. Iterating $(224)$ we obtain

$$
\frac{f^{\prime}\left(\zeta_{0}+n\right)}{f\left(\zeta_{0}+n\right)}=\prod_{\ell=0}^{n-1}\left[\frac{1}{S^{\prime}\left(f\left(\zeta_{0}+1+\ell\right)\right)} \frac{f\left(\zeta_{0}+\ell\right)}{S^{-1}\left(f\left(\zeta_{0}+\ell\right)\right)}\right] \frac{f^{\prime}\left(\zeta_{0}\right)}{f\left(\zeta_{0}\right)}
$$

The product in (225) can be bounded as

$$
\prod_{\ell=0}^{n-1}\left[C f\left(\zeta_{0}+\ell\right)\right]
$$

and taking the logarithm of this expression and taking into account that $\frac{f(\zeta+1)}{f(\zeta)} \geq 2$ for $\zeta$ large enough we obtain, after adding a geometric series, an upper estimate for the product in (226) of the form

$$
\exp \left(B \log \left(f\left(\zeta_{0}+n-1\right)\right)\right)=\left(f\left(\zeta_{0}+n-1\right)\right)^{B}
$$

for some $B>0$, whence (225) yields

$$
f^{\prime}(\zeta) \leq C f(\zeta)(f(\zeta-1))^{B}
$$

and since (134) implies that $f(\zeta-1) \leq C \log (f(\zeta))$ we obtain (220) for $k=1$. The proof of (220) for $k=2,3$ is similar.

In order to show (222) we iterate (134) to compute $f\left(\zeta+\frac{C}{f(\zeta)}\right)$

$$
f\left(\zeta+\frac{C}{f(\zeta)}\right)=S^{-1}\left(S^{-1}\left(\ldots S^{-1}\left(f\left(\zeta+\frac{C}{f(\zeta)}-n\right)\right)\right)\right)
$$

where the number of iterations $n$ is such that $\zeta+\frac{C}{f(\zeta)}-n \in\left[\zeta_{0}, \zeta_{0}+1\right]$. Since $f(\zeta)$ is huge we can approximate the terms $S^{-1}\left(f\left(\zeta+\frac{C}{f(\zeta)}-n\right)\right)$ as $S^{-1}(f(\zeta-) n)+\frac{C\left(S^{-1}\right)^{\prime}(f(\zeta-n))}{f(\zeta)}$. Using this approximation in $n-1$ iterations, as well as (223) we obtain the approximation

$$
f\left(\zeta+\frac{C}{f(\zeta)}\right)=S^{-1}\left(f(\zeta-1)+\frac{C f^{\prime}(\zeta-1)}{f(\zeta)}\right)
$$

and using (227), (222) follows. A more rigorous proof would just replace the approximation using derivatives by upper bounds. Similar approximations might be derived for the derivatives.

\section{APPENDIX C: STABILITY OF SOME CONVOLUTION OP- ERATORS}

In this Appendix we study the stability properties of the operators associated to times $\tau$ of order one in the equations (200) and (210). To this end, we use the fact that the dominant operators in this time scale are convolution operators that can be studied using Laplace transforms. 


\subsection{STABILITY FOR THE TRANSITION PROBLEM}

Using the time variable $\tau=f(\bar{\zeta}+1)$ we can transform (200), neglecting some small terms that are introduced in the error term as

$$
\begin{aligned}
& \frac{1}{(\alpha-1)^{2}} \frac{d^{2} \lambda(\tau)}{d \tau^{2}}-\Omega^{\prime}\left(-F_{\text {int }}(\alpha)\right) \frac{d \lambda(\tau)}{d \tau}+\Omega^{\prime \prime}\left(-F_{\text {int }}(\alpha)\right) \lambda(\tau)- \\
& -\int_{\tau_{0}}^{\tau} \Omega^{\prime \prime \prime}\left(s-\tau-F_{\text {int }}(\alpha)\right) \lambda(s) d s+H(\tau) \\
& =O\left(\frac{1}{(f(\bar{\zeta}))^{\alpha}}\right) \equiv r(\tau)
\end{aligned}
$$

where $H(\tau)$ contains the contribution of the term $\int_{\zeta_{0}}^{\bar{\zeta}+\delta_{1}(\bar{\zeta})} f^{\prime}(\zeta) \Psi^{\prime \prime \prime}(f(\zeta)-f(\bar{\zeta})) \lambda(\zeta) d \zeta$ that might be approximated to the leading order as $C \lambda(S(\tau))$. Therefore, this term varies, for $\tau \rightarrow \infty$ in a longer time scale that the other terms in (228) and, as a consequence, if the convolution part of the operator can be shown to yield convergence, it would be possible to approximate (200) as (194). Taking the Laplace transform of (228) that we define as

$$
\tilde{f}(z)=\int_{0}^{\infty} f(\tau) e^{-\tau z} d \tau
$$

we obtain

$$
\tilde{\lambda}(z)=\frac{\tilde{H}(z)+\tilde{r}(z)+C_{1} z+C_{2}}{\frac{z^{2}}{(\alpha-1)^{2}}-\Omega^{\prime}\left(-F_{\mathrm{int}}(\alpha)\right) z+\Omega^{\prime \prime}\left(-F_{\mathrm{int}}(\alpha)\right)-\omega(z)}
$$

where

$$
\omega(z, \alpha)=\int_{0}^{\infty} \Omega^{\prime \prime \prime}\left(-\tau-F_{\text {int }}(\alpha)\right) e^{-\tau z} d \tau
$$

Standard properties of the Laplace transform would show that the solutions of (228) approach to a constant value for $1<<\tau$ if the function

$$
\Phi(z, \alpha)=\frac{z^{2}}{(\alpha-1)^{2}}-\Omega^{\prime}\left(-F_{\text {int }}(\alpha)\right) z+\Omega^{\prime \prime}\left(-F_{\text {int }}(\alpha)\right)-\omega(z, \alpha)
$$

does not have zeroes in the half-plane $\{\operatorname{Re}(z)>0\}$. (A similar argument, in a fully rigorous manner can be found in [14]). We have studied the zeroes of the function $\Phi(z)$ using the argument principle for the particular value $\alpha=\frac{1}{2}$. After some computations the function $\Phi\left(z ; \frac{1}{2}\right)$ becomes:

$$
\Phi\left(z ; \frac{1}{2}\right)=4.0 z^{2}+1.9024 z+0.23968-2 e^{z F_{\mathrm{int}}(\alpha)} I(z)
$$

where

$$
F_{\text {int }}(\alpha)=F_{\text {int }}\left(\frac{1}{2}\right)=1.6622
$$

and

$$
\begin{aligned}
& I(z) \\
& =\int_{\left(\frac{1}{2}\right)^{1 / 3}}^{1} \frac{\left(-4-17 Y+12 Y^{6}-29 Y^{3}-45 Y^{2}+60 Y^{5}+68 Y^{4}\right)}{\left(Y^{2}+Y+1\right)^{5} Y^{3}} . \\
& \exp \left(-z\left(\frac{4}{3} \log \left(1+\frac{Y}{2}\right)-\frac{Y}{(Y-1)}+\frac{5}{3} \log (1-Y)\right)\right) d Y
\end{aligned}
$$


In Figures 1,2 we describe the image of a contour surrounding the half-plane $\{\operatorname{Re}(z)>0\}$ for a large value of $R \rightarrow \infty$ and its image by means of this function. More precisely, Figure 1 contains the image of the vertical segment $[0,10 i]$ by the function $\Phi\left(z ; \frac{1}{2}\right)$. The second picture provides a detail of the same curve near the origin. On the other hand Figure 2 shows the image of the half-circle $10 e^{i \theta}, \theta \in\left[0, \frac{\pi}{2}\right]$ by means of $\Phi\left(z ; \frac{1}{2}\right)$. The second picture provides a detail of this curve for large values of $|z|$. The image by means of $\Phi\left(z ; \frac{1}{2}\right)$ of the segment $[-10 i, 0]$ and the half-circle $10 e^{i \theta}, \theta \in\left[-\frac{\pi}{2}, 0\right]$ can be obtained by reflection with respect to the real axis, since $\left(\Phi\left(z ; \frac{1}{2}\right)\right)^{*}=\Phi\left(z^{*} ; \frac{1}{2}\right)$. It follows from these pictures that the image by means of $\Phi\left(z ; \frac{1}{2}\right)$ of the curve $[-10 i, 10 i] \cup\left\{z=10 e^{i \theta}: \theta \in\left[\frac{\pi}{2},-\frac{\pi}{2}\right]\right\}$ does not surround the origin, and therefore, the argument principle implies that there are not zeroes of $\Phi\left(z ; \frac{1}{2}\right)$ in the half-plane $\{\operatorname{Re}(z) \geq 0\}$. This yields the desired stability.
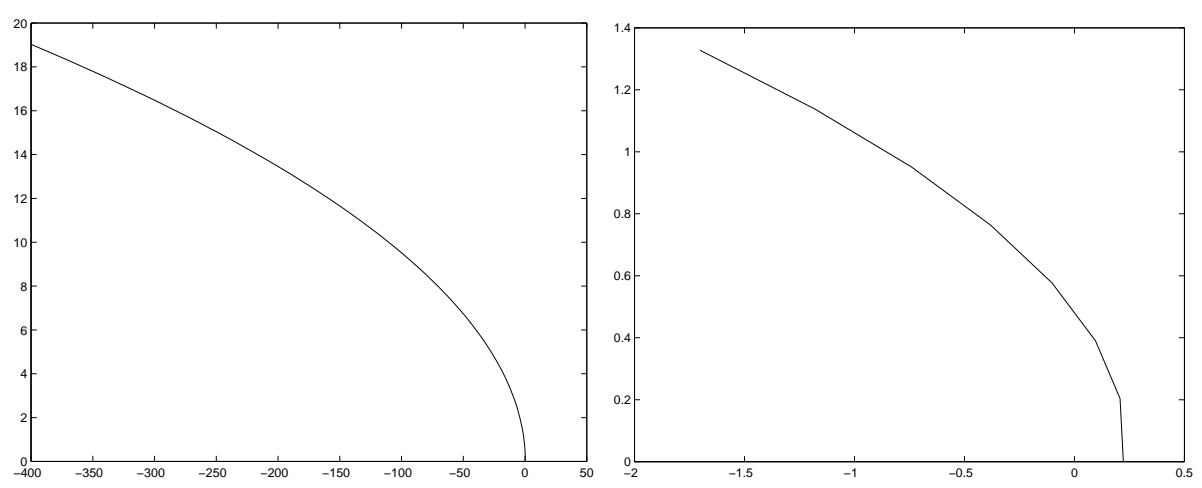

Figure 1
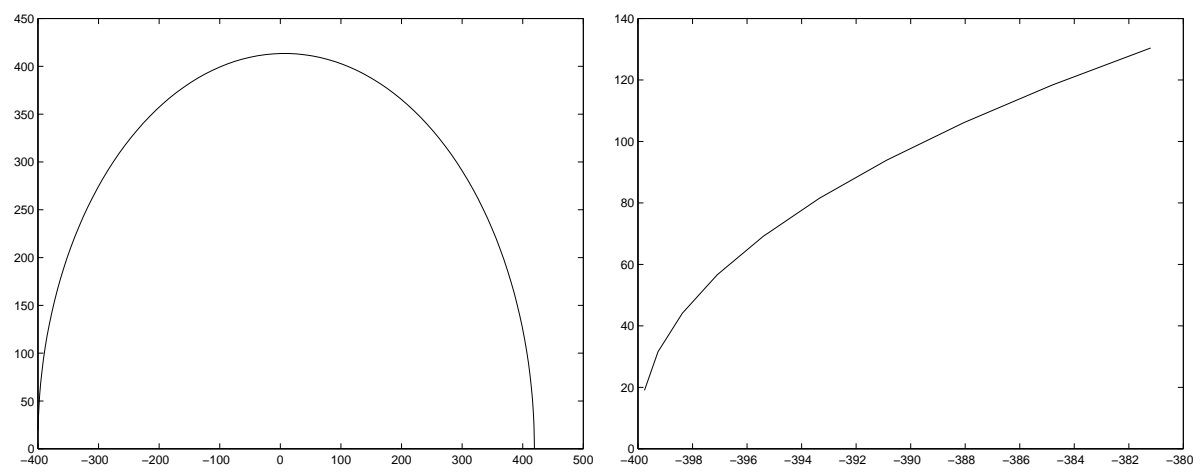

Figure 2 


\subsection{STABILITY FOR THE LINEARIZED LSW MODEL}

In the case of the linearization of the whole LSW model the study can be made in a similar manner. Using the time variable $\tau$, equation (210) becomes, neglecting the smallest terms

$$
\begin{aligned}
& \left(\int_{0}^{1} \frac{e^{-F_{\mathrm{int}}(W)} d W}{(W-1)^{2}}\right) \frac{d^{2} \lambda(\tau)}{d \tau^{2}}-\left(\int_{0}^{1} e^{-F_{\mathrm{int}}(W)} \Omega^{\prime}\left(-F_{\mathrm{int}}(W)\right) d W\right) \frac{d \lambda(\tau)}{d \tau} \\
& +\left(\int_{0}^{1} e^{-F_{\mathrm{int}}(W)} \Omega^{\prime \prime}\left(-F_{\mathrm{int}}(W)\right) d W\right) \lambda(\tau) \\
& -\int_{\tau_{0}}^{\tau}\left[\int_{0}^{1} e^{-F_{\mathrm{int}}(W)} \Omega^{\prime \prime \prime}\left(s-\tau-F_{\mathrm{int}}(W)\right) d W\right] \lambda(s) d s+H(\tau)+r(\tau)
\end{aligned}
$$

where as in (228), $r(\tau)$ converges to zero and $H(\tau)$ varies slowly in the time scale $S(\tau)$.

Equation (229) can be studied similarly to $(228)$ using Laplace transforms. The stability properties of the convolution operator (including the derivatives) reduces then to prove that the function

$$
\Psi(z)=\int_{0}^{1} \Phi(z, W) e^{-F_{\text {int }}(W)} d W
$$

does not have zeroes in the half-plane $\{\operatorname{Re}(z)>0\}$. This property can be shown exactly as in the previous Subsection using the argument principle. Figure 3 shows the image of the segment $[0,10 i]$. The second picture provides a detail of the region close to the origin. Figure 4 shows the image by $\Psi$ of the half-circle $10 e^{i \theta}, \theta \in\left[0, \frac{\pi}{2}\right]$. The second picture gives a detail of the region or large values of $|z|$.
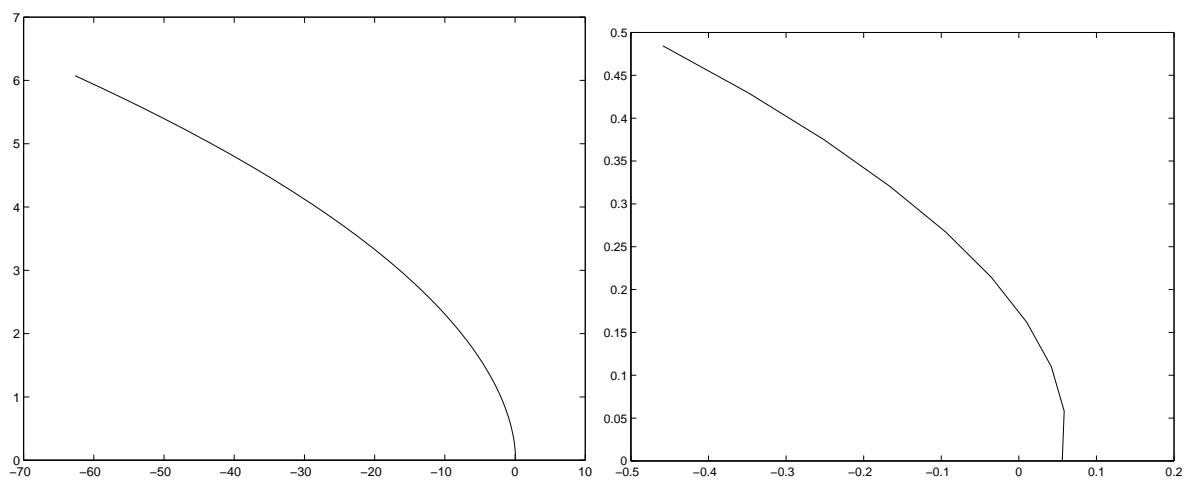

Figure 3
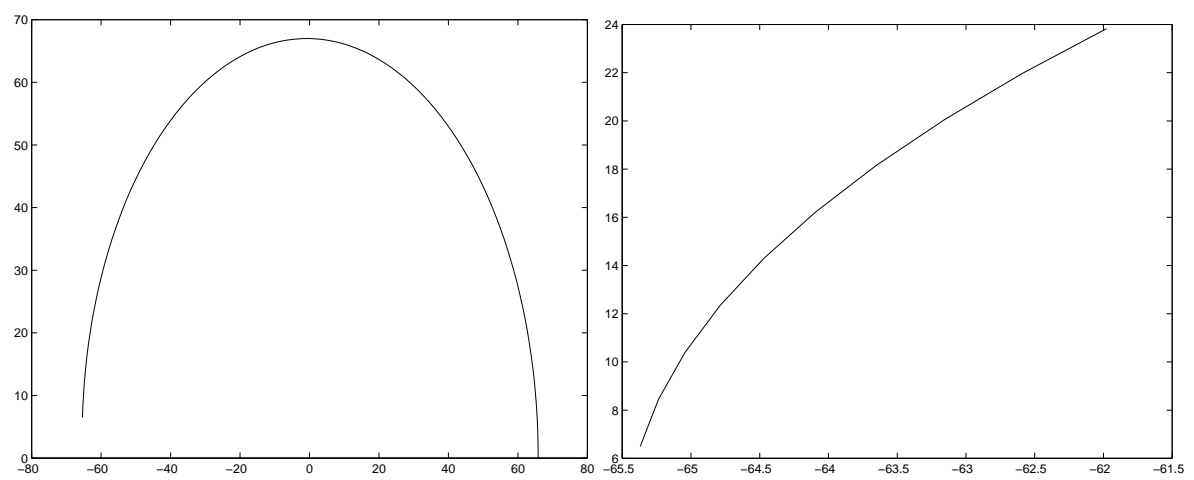
Figure 4

\section{APPENDIX D: A HEURISTIC LINEARIZATION OF THE LSW MODEL}

In this Appendix we sketch the main ideas of an alternative linearization of the LSW model, that is less rigorous and precise that the one in Subsection 4.5, but provides a simpler intuition on the two time scale character of the linearized problem that the one based in the functions $f(\zeta)$. We assume in all this Section that $S(\tau) \sim a \log (\tau)$ as $\tau \rightarrow \infty$.

Arguing as in Subsection 4.5 we can derive the equation (204). Let us write

$$
\beta(\tau)=\tilde{\beta}(\tau)+\sigma(\tau)
$$

where $\tilde{\beta}(\tau)$ is the solution of (174)-(176).

We look for solutions of (111)-(112) in the form

$$
W\left(s ; W_{0}(\tau ; W)\right)=\bar{W}(s ; \theta(s ; \tau ; W))
$$

Then $\theta(s ; \tau ; W)$ satisfies:

$$
\begin{aligned}
\theta_{s}(s ; \tau ; W) & =\frac{3 \sigma(s)(\bar{W}(s ; \theta(s ; \tau ; W)))^{1 / 3}}{\frac{\partial \bar{W}(s ; \theta(s ; \tau ; W))}{\partial \bar{W}_{0}}} \\
\theta(\tau ; \tau ; W) & =\bar{W}_{0}(\tau ; W)
\end{aligned}
$$

On the other hand, using (231) we obtain:

$$
\theta(0 ; \tau ; W)=W_{0}(\tau ; W)
$$

Integrating (232) with the conditions (233), (234) we obtain:

$$
W_{0}(\tau ; W)-\bar{W}_{0}(\tau ; W)=-\int_{0}^{\tau} \frac{3 \sigma(s)(\bar{W}(s ; \theta(s ; \tau ; W)))^{1 / 3}}{\frac{\partial \bar{W}(s ; \theta(s ; \tau ; W))}{\partial W_{0}}} d s
$$

Using (235) we obtain, arguing as in Subsection 4.5 we can approximate (204) to the leading order by the following integral equation

$$
\int_{0}^{\tau} K(\tau, s) \sigma(s) d s=\varepsilon(\tau)
$$

where $\lim _{\tau \rightarrow \infty} \varepsilon(\tau)=0$, and

$$
K(\tau, s)=\frac{v_{0} e^{-\tau}}{\int_{0}^{1} e^{-F_{\mathrm{int}}(W)} d W} \int_{0}^{1} \frac{e^{-F_{\mathrm{int}}(W)}(\bar{W}(s ; \theta(s ; \tau ; W)))^{1 / 3}}{\bar{W}_{0}(\tau ; W) \lambda\left(w_{\mathrm{ext}}\left(\bar{W}_{0}(\tau ; W)\right)\right) \frac{\partial \bar{W}}{\partial W_{0}}(s ; \theta(s ; \tau ; W))} d W
$$

We can approximate $\bar{W}$ using (96) if $|W-1|$ is of order one. On the other hand, in the region $W \approx 1$, using the fact that $\tilde{\beta}(\tau)$ has the asymptotics

$$
\tilde{\beta}(\tau) \sim-\frac{1}{4}\left[\frac{1}{\tau^{2}}+\frac{1}{\tau^{2}(\log (\tau))^{2}}+\frac{1}{\tau^{2}(\log (\tau))^{2}(\log \log (\tau))^{2}}+\ldots\right] \text { as } \tau \rightarrow \infty
$$


it follows, after some computations, that

$$
\bar{W}\left(s ; \bar{W}_{0}(0, \tau)\right)-1 \sim \frac{1}{s}\left(\frac{3}{2}+\frac{3}{\log \left(\frac{s}{\tau}\right)}\right)
$$

for $\tau-s>>1, s>>1$. Differentiating (236) with respect to $\tau$, and using the approximations (116), (122) we obtain the following equation

$$
\sigma(\tau)+\int_{0}^{\tau} Q(\tau, s) \sigma(s) d s=v(\tau)
$$

where $\lim _{\tau \rightarrow \infty} v(\tau)=0$, and where the asymptotics of $Q(\tau, s)$ is given by

$$
Q(\tau, s) \sim A s \log \left(\frac{s}{\tau}\right)\left[\log \left(\frac{s}{\tau}\right)-2\right]
$$

for $(\tau-s) \approx \tau$ and $\tau>>1$, where

$$
A \equiv \frac{\int_{0}^{1} e^{-F_{\mathrm{int}}(W)} d W}{3 \int_{0}^{1} \frac{e^{-F_{\mathrm{int}}(W)}(W)^{1 / 3}}{2-3 W^{1 / 3}+W} d W}
$$

On the other hand

$$
Q(\tau, s) \sim \Phi(\tau-s)=\frac{\partial K(\tau-s)}{\partial \tau}
$$

as $\tau>>1$, for $\tau-s$ of order one, where

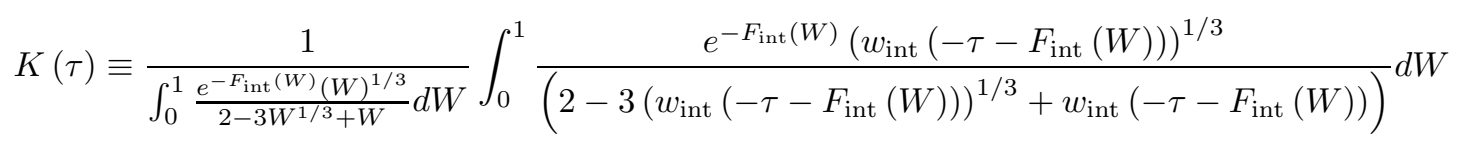

We remark that the asymptotics (241), (242) match in the intermediate region $1<<(\tau-s)<<\tau$.

The integral equation (240) has two natural time scales, namely $(\tau-s) \approx 1$ and $s \approx \log (\tau)$. This equation might be analyzed in the same manner as equation (210) was studied in Subsection 4.5 decoupling both time scales. In particular, the fastest time scale is the associated to times $(\tau-s) \approx 1$. In this time scale the equation (240) might be approximated by a convolution equation that can be solved using Laplace transforms. More precisely, the integral equation

$$
\sigma(\tau)+\int_{0}^{\tau} \Phi(\tau-s) \sigma(s) d s=v(\tau)
$$

can be inverted. as

$$
\sigma(\tau)=v(\tau)-\int_{0}^{\tau} G(\tau-s) v(s) d s
$$

where

$$
|G(\tau)| \leq C \min \left\{1, \frac{|\log (\tau)|}{\tau^{4}}\right\} \text { for } \tau \geq 2
$$

and

$$
\int_{0}^{\infty} G(\tau) d \tau-1=\int_{0}^{\infty} \tau G(\tau) d \tau=0
$$

We can then exploit the separations of scales of the problem as follows. For any given $\tau_{1}>0$ large, we rewrite (240) in the form:

$$
\sigma(\tau)+\int_{\tau_{1}}^{\tau} Q(\tau, s) \sigma(s) d s=\sigma\left(\tau_{1}\right)+\left[v(\tau)-v\left(\tau_{1}\right)\right]-\int_{0}^{\tau_{1}}\left[Q(\tau, s)-Q\left(\tau_{1}, s\right)\right] \sigma(s) d s
$$


If $\left(\tau-\tau_{1}\right)=O(1)$ we can use the inversion formula (244) and rewrite this equation as:

$$
\begin{aligned}
\sigma(\tau) & =\sigma\left(\tau_{1}\right)+\left[v(\tau)-v\left(\tau_{1}\right)\right]-\int_{0}^{\tau_{1}}\left[Q(\tau, s)-Q\left(\tau_{1}, s\right)\right] \sigma(s) d s- \\
& -\sigma\left(\tau_{1}\right) \int_{\tau_{1}}^{\tau} G(\tau-s) d s-\int_{\tau_{1}}^{\tau} G(\tau-s)\left[v(s)-v\left(\tau_{1}\right)\right] d s \\
& +\int_{\tau_{1}}^{\tau} G(\tau-s)\left(\int_{0}^{\tau_{1}}\left[Q(s, \xi)-Q\left(\tau_{1}, \xi\right)\right] \sigma(\xi) d \xi\right) d s
\end{aligned}
$$

After some integrations by parts, (247) becomes

$$
\begin{aligned}
& \sigma(\tau)-\int_{0}^{\tau_{1}}\left(\int_{\tau_{1}}^{\tau} W(\tau-s) \frac{\partial^{2}}{\partial \tau^{2}}(Q(s, \xi)) d s\right) \sigma(\xi) d \xi \\
& =-\sigma\left(\tau_{1}\right) W^{\prime}\left(\tau-\tau_{1}\right)+\mu\left(\tau, \tau_{1}\right)+W\left(\tau-\tau_{1}\right) \int_{0}^{\tau_{1}} \frac{\partial}{\partial \tau}\left[Q\left(\tau_{1}, \xi\right)\right] \sigma(\xi) d \xi
\end{aligned}
$$

where

$$
W(\tau)=\int_{0}^{\tau}(\tau-\xi) G(\xi) d \xi-\tau
$$

and $\mu\left(\tau, \tau_{1}\right)$ approaches zero uniformly for $\tau \geq \tau_{1}, \tau_{1} \rightarrow \infty$. At this stage we use the following key approximation. The function $Q(s, \xi)$ is approximately constant if $s$ changes amounts of order one and $(s-\xi)>>1$. Since $W$ varies in time scales of order one and $|W(\tau)| \leq \frac{C|\log (\tau)|}{\tau^{2}}$ for $\tau \geq 2$ we have the approximation

$$
\int_{\tau_{1}}^{\tau} W(\tau-s) \frac{\partial^{2}}{\partial \tau^{2}}(Q(s, \xi)) d s \approx\left(\int_{-\infty}^{0} W(-s) d s\right) \frac{\partial^{2}}{\partial \tau^{2}}(Q(s, \xi))
$$

for $1<<(s-\xi)<<s,\left(\tau-\tau_{1}\right)>>1$. Using then (242) it follows after some computations that

$$
\sigma(\tau)+\frac{4}{3} \int_{0}^{\tau_{1}}\left(\frac{s}{\tau^{2}} \log \left(\frac{s}{\tau}\right) \sigma(s) d s\right)=\hat{\varepsilon}(\tau)
$$

where we have replaced $\tau_{1}$ by $\tau$, and where $\lim _{\tau \rightarrow \infty} \hat{\varepsilon}(\tau)=0$. Equation (249) approximates the LSW model in the slower time scale $\log (\tau)$, and it is the analogous of (211). This integral equation is homogeneous with respect to the rescaling $s \rightarrow \lambda s, \tau \rightarrow \lambda \tau$ and, as a consequence, it can be solved using Mellin's transforms. The final formula for the solution is

$$
\sigma(\tau)=\int_{0}^{\tau} \Psi(s, a) \varepsilon(s) d s=\hat{\varepsilon}(\tau)-\frac{2}{\sqrt{3}} \int_{0}^{\tau} \hat{\varepsilon}(a) \frac{a}{\tau^{2}} \sin \left(\frac{2}{\sqrt{3}} \log \left(\frac{\tau}{a}\right)\right) d a
$$

In particular (250) implies that $\lim _{\tau \rightarrow \infty} \sigma(\tau)=0$, and this yields the desired stability.

The argument above provides an intuitive explanation of the onset of two time scales in the problem. However, it is not completely precise because the asymptotics (239) ignores the presence of higher corrective terms of the form $\log \log \left(\frac{s}{\tau}\right)$, and similar ones. Nevertheless, and since the argument in this Appendix is more intuitive that the one in Subsection 4.5 a sketch of it has been given. 